\title{
ROOT NUMBERS AND PARITY OF RANKS OF ELLIPTIC CURVES
}

\author{
TIM AND VLADIMIR DOKCHITSER \\ to J.W.S. Cassels on the occasion of his 87 th birthday
}

\begin{abstract}
The purpose of the paper is to complete several global and local results concerning parity of ranks of elliptic curves. Primarily, we show that the Shafarevich-Tate conjecture implies the parity conjecture for all elliptic curves over number fields, give a formula for local and global root numbers of elliptic curves and complete the proof of a conjecture of Kramer and Tunnell in characteristic 0. The method is to settle the outstanding local formulae by deforming from local fields to totally real number fields and then using global parity results.
\end{abstract}

\section{Contents}

1. Introduction

2. 2-parity over totally real fields

3. Continuity of local invariants

4. The Kramer-Tunnell conjecture

5. Elliptic curves with a $p$-isogeny

6. Parity for twists coming from Brauer relations

7. Parity over fields and the global root number formula

8. The local root number formula

References

\section{INTRODUCTION}

The principal arithmetic invariant of an elliptic curve $E$ defined over a number field $K$ is its Mordell-Weil $\operatorname{rank} \operatorname{rk} E / K$, the rank of the group of rational points modulo torsion. In view of the Birch-Swinnerton-Dyer conjecture, its parity should be governed by another arithmetic invariant, the global root number $w(E / K)= \pm 1$ (the expected sign in the conjectural functional equation for the $L$-function of $E / K)$ :

Conjecture 1.1 (Parity Conjecture). $(-1)^{\mathrm{rk} E / K}=w(E / K)$.

Date: June 9, 2009.

2000 Mathematics Subject Classification. Primary 11G05; Secondary 11G07, 11G40. 
The purpose of this paper is to clean up some known results concerning this conjecture. Primarily, we show that it follows from finiteness of the Tate-Shafarevich group, and give an explicit formula for $w(E / K)$ and thus, conjecturally, for the parity of the rank:

Theorem 1.2 (=Thm. 7.1). Let $E$ be an elliptic curve over a number field $K$, and suppose $\amalg(E / K(E[2]))$ has finite 2- and 3-primary parts. Then

$$
(-1)^{\mathrm{rk} E / K}=w(E / K) \text {. }
$$

Theorem 1.3 (=Thm. 7.2). Let $E$ be an elliptic curve over a number field $K$, and set $F=K(E[2])$ and $d=[F: K]$. Choose a non-trivial 2-torsion point $P$ of $E$, defined over $K$ if $d \leq 2$, and write $E^{\prime}=E /\{O, P\}$ for the 2-isogenous curve over $L=K(P)$. Then

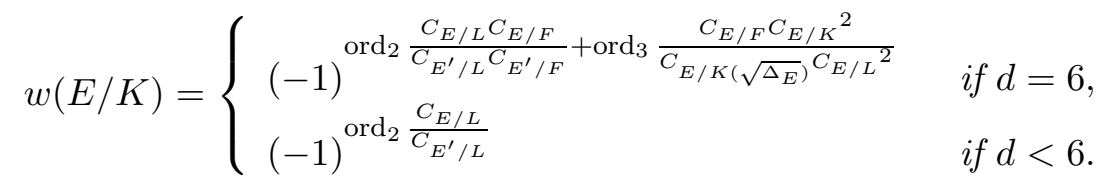

Here $\Delta_{E}$ is the discriminant of $E / K$, and $C_{E / K}$ the product 1 of the 'local fudge factors' and periods in the conjectural Birch-Swinnerton-Dyer formula for $L(E / K, s)$ at $s=1$, see $\$ 1.3$ for the precise definition.

Theorem 1.2 is already known under mild technical restrictions on $E$ (4] Thm. 1.3). We remove the restrictions by a roundabout approach that avoids unpleasant local computations at the troublesome primes above 2 and 3. This method of dealing with difficult reduction cases applies to other parity-related local formulae. In particular, we will use it to derive a formula for local root numbers (analogous to Theorem 1.3) and to prove the remaining case of the Kramer-Tunnell conjecture for elliptic curves over $p$-adic fields.

Most of these local formulae have a global counterpart related to Selmer groups. Recall that there is a version of the parity conjecture for the $p^{\infty}$-Selmer rank $\mathrm{rk}_{p} E / K$, the $\mathbb{Z}_{p}$-corank of the $p^{\infty}$-Selmer group:

Conjecture 1.4 ( $p$-Parity Conjecture). $(-1)^{\mathrm{rk}_{p} E / K}=w(E / K)$.

It is of course expected that $\operatorname{rk}_{p} E / K=\operatorname{rk} E / K$ for each $p$, as the difference is the number of copies of $\mathbb{Q}_{p} / \mathbb{Z}_{p}$ in the (supposedly finite) group $\amalg(E / K)$. The cases that we address are 2-parity over quadratic extensions, $p$-parity for curves with a $p$-isogeny when $p=2$ and 3 , and a slight improvement on [6] for $p$-parity results coming from Brauer relations and in $p$-adic towers. See $\$ 1.2$ for precise statements.

\footnotetext{
${ }^{1}$ Computational note: $C_{E / K}=\prod_{v} C\left(E / K_{v}, \omega\right)$ for any invariant differential $\omega$ for $E / K$. At finite places, $C\left(E / K_{v}, \omega\right)=c_{v} \cdot\left|\omega / \omega_{v}^{\circ}\right|_{v}$, where $c_{v}$ is the local Tamagawa number at $v$ and $\omega_{v}^{\circ}$ the minimal differential at $v$, and hence can be computed with Tate's algorithm. The contributions from $v \mid \infty$ cancel in the ord ${ }_{3}$ term when using any $\omega$ for $E / K$. In the ord 2 term, use an $L$-rational $\omega^{\prime}$ for $E^{\prime}$ and $\omega=\phi^{*} \omega^{\prime}$ for $E$, where $\phi: E \rightarrow E^{\prime}$ is the 2-isogeny; then every $v \mid \infty$ where $\phi$ maps $E\left(L_{v}\right)$ onto $E^{\prime}\left(L_{v}\right)$ contributes -1 to the $\operatorname{ord}_{2}$ term.
} 
1.1. The method \& the Kramer-Tunnell conjecture. There are several results that express the parity of a specific Selmer rank in terms of purely local data of the elliptic curve (e.g. [2, 4, 5, 9, 15, 17]). Now recall that the global root number is defined as the product over all places of local root numbers,

$$
w(E / K)=\prod_{v} w\left(E / K_{v}\right)
$$

Hence, if this 'local data' can be related to the corresponding local root number, it can be used to prove the parity conjecture for this Selmer rank.

For example, Kramer's theorem [15] Thm. 1 asserts that for a quadratic extension $F=K(\sqrt{\alpha})$,

$$
(-1)^{\mathrm{rk}_{2} E / F}=\prod_{v}(-1)^{\operatorname{dim}_{\mathbb{F}_{2}} \frac{E\left(K_{v}\right)}{N E\left(F_{w}\right)},}
$$

the product taken over all places $v$ of $K$, choosing any $w \mid v$ of $F$. Here $N: E\left(F_{w}\right) \rightarrow E\left(K_{v}\right)$ is the norm map on points. In [16] Kramer and Tunnell found the conjectural relation to the root number,

$$
(-1)^{\operatorname{dim}_{\mathbb{F}_{2}} \frac{E\left(K_{v}\right)}{N E\left(F_{w}\right)}}=w\left(E / K_{v}\right) w\left(E_{\alpha} / K_{v}\right)\left(-\Delta_{E}, \alpha\right)_{K_{v}},
$$

where $E_{\alpha}$ is the quadratic twist of $E$ by $\alpha$, and $(\cdot, \cdot)$ is the Hilbert symbol. Granting this relation and taking the product over all $v$, we get the 2-parity conjecture for $E / F$,

$$
(-1)^{\mathrm{rk}_{2} E / F}=w(E / F) .
$$

(Observe that the Hilbert symbols disappear globally by the product formula.) Kramer and Tunnell established (因) in all cases but the most horrible one, namely when $v \mid 2, F_{w} / K_{v}$ is ramified and $E / K_{v}$ has additive reduction.

We now explain how to prove (囝) in the remaining cases (see $\$ 4$ for details). Instead of confronting residue characteristic 2, we use a 'global-to-local' approach. The point is that the $p$-parity conjecture can be seen to hold over totally real fields for all elliptic curves with non-integral $j$-invariant, by considerations that exploit modularity (Nekováŕ 20 for odd $p$ and Theorem 2.4 for $p=2$ ). Therefore we can prove the local formula by reversing the argument above: if $K$ and $F$ are totally real and we know that (因) holds at all places $v$ but one, it must hold over the remaining place as well. Because there is a sufficient supply of such fields and curves and all the terms in (因) are continuous (as functions of the coefficients of the curve), it follows that the local formula must always be true.

To summarise, we obtain:

Theorem 1.5 (Kramer-Tunnell conjecture in characteristic 0). Let $\mathcal{K}$ be a local field of characteristic zero, $\mathcal{F}=\mathcal{K}(\sqrt{\alpha})$ a quadratic extension, $E / \mathcal{K}$ an elliptic curve and $E_{\alpha} / \mathcal{K}$ its quadratic twist by $\alpha$. Then

$$
w(E / \mathcal{K}) w\left(E_{\alpha} / \mathcal{K}\right)\left(-\Delta_{E}, \alpha\right)_{\mathcal{K}}=(-1)^{\operatorname{dim}_{\mathbb{F}_{2}} \frac{E(\mathcal{K})}{N E(\mathcal{F})}} .
$$

Here $\Delta_{E}$ is the discriminant of some model of $E / \mathcal{K}, N: E(\mathcal{F}) \rightarrow E(\mathcal{K})$ the norm map on points, and $(\cdot, \cdot)_{\mathcal{K}}$ the Hilbert symbol in $\mathcal{K}$. 
Corollary 1.6 (2-parity in quadratic extensions). Let $E$ be an elliptic curve over a number field $K$. For every quadratic extension $F / K$,

$$
(-1)^{\mathrm{rk}_{2} E / F}=w(E / F) .
$$

1.2. Other parity results. The following two theorems are slight extensions of known results for general $p$ (see 92 and $\$ 5$ ).

Theorem 1.7 (see Thm. 2.4). Let $K$ be a totally real field, and $E / K$ an elliptic curve with non-integral $j$-invariant. Then the 2-parity conjecture holds for $E / K$.

Theorem 1.8 (=Cor. 5.8). Let $E$ be an elliptic curve over a number field $K$ that admits a K-rational isogeny of degree $p=2$ or 3. Then

$$
(-1)^{\mathrm{rk}_{p} E / K}=w(E / K) \text {. }
$$

Next, we consider twists of elliptic curves by Artin representations. Recall that if $F / K$ is a Galois extension, the dual $p^{\infty}$-Selmer

$$
\mathcal{X}_{p}(E / F)=\left(\text { Pontryagin dual of the } p^{\infty} \text {-Selmer group of } E / F\right) \otimes \mathbb{Q}_{p}
$$

is a $\mathbb{Q}_{p}$-rational representation of $G=\operatorname{Gal}(F / K)$. One can compare multiplicities $\left\langle\tau, \mathcal{X}_{p}(E / F)\right\rangle$ of $G$-representations in it to the twisted root numbers $w(E / K, \tau)$; conjecturally,

$$
(-1)^{\left\langle\tau, \mathcal{X}_{p}(E / F)\right\rangle}=w(E / K, \tau) \quad(p \text {-parity conjecture for twists), }
$$

for every self-dual complex representation $\tau$ of $G$. When $\tau=\mathbb{C}[G / H]$, this simply recovers the $p$-parity conjecture for $E / F^{H}$.

Our main result concerning twists is somewhat technical (Corollary 6.8), but here are a few consequences (see \$6.3):

Theorem 1.9. For every elliptic curve $E / \mathbb{Q}$ and number field $L \subset \mathbb{Q}\left(E\left[3^{\infty}\right]\right)$,

$$
(-1)^{\mathrm{rk}_{3} E / L}=w(E / L) .
$$

Theorem 1.10. Let $p$ be an odd prime, and $m, n \geq 1$ integers. For every elliptic curve $E / \mathbb{Q}$ and number field $F \subset \mathbb{Q}\left(\mu_{p^{n}}, \sqrt[p]{m}\right)$,

$$
(-1)^{\mathrm{rk}_{p} E / F}=w(E / F) \text {. }
$$

Theorem 1.11. Let $p \equiv 3 \bmod 4$ be a prime number and $E / \mathbb{Q}$ an elliptic curve. Suppose $F$ is a p-extension of an abelian extension of $\mathbb{Q}$, Galois over $\mathbb{Q}$. Then

$$
(-1)^{\left\langle\tau, \mathcal{X}_{p}(E / F)\right\rangle}=w(E, \tau)
$$

for every orthogonal representation $\tau$ of $\operatorname{Gal}(F / \mathbb{Q})$; in other words, the p-parity conjecture holds for all orthogonal twists, and in particular over all subfields of $F$.

Finally, we turn to local root numbers of elliptic curves. The definition of these is not constructive, but they have been classified in many cases. For instance, $w\left(E / K_{v}\right)=-1$ when $v$ is Archimedean or when $E / K_{v}$ has 
split multiplicative reduction, and $w\left(E / K_{v}\right)=+1$ when $E / K_{v}$ has good or non-split multiplicative reduction. Thus, if $E / K$ is semistable,

$$
w(E / K)=(-1)^{\#\{v \mid \infty \text { in } K\}+\#\{v \text { split mult. for } E / K\}} \text {. }
$$

At places of additive reduction, an explicit classification has been given by Rohrlich [21] for $v \nmid 2,3$, Halberstadt [11] for $K=\mathbb{Q}$, and Kobayashi [14] for $v \mid 3$. These express the root number in terms of the standard invariants of $E / K$, making it easy to compute, as in the semistable case. When $v \mid 2$, the existing formulae for $w\left(E / K_{v}\right)([30,7])$ are complicated and harder to use, as they rely on epsilon-factors of Galois characters.

From the proof of the global root number formula of Theorem 1.3 we will extract a uniform formula for the local root numbers. It is slightly more cumbersome to state as it involves all three 2-isogenous curves:

Theorem 1.12 (see $\$ 8$ ). Let $E$ be an elliptic curve over a finite extension $\mathcal{K}$ of $\mathbb{Q}_{p}$. Fix an invariant differential $\omega$ for $E / \mathcal{K}$ and write

$$
H_{l}^{E / \mathcal{K}}= \begin{cases}+1 & \text { if } C(E / \mathcal{K}, \omega) \text { has even } l \text {-adic valuation, } \\ -1 & \text { if } C(E / \mathcal{K}, \omega) \text { has odd } l \text {-adic valuation. }\end{cases}
$$

Denote $\mathcal{F}=\mathcal{K}(E[2])$ and $d=[\mathcal{F}: \mathcal{K}]$. Let $E^{\prime}, E^{\prime \prime}$ and $E^{\prime \prime \prime}$ be the three curves 2-isogenous to $E$, defined over suitable intermediate fields $\mathcal{L}^{\prime}, \ldots$ of $\mathcal{F} / \mathcal{K}$; when $d=2$ we select $E^{\prime}$ to be the one defined over $\mathcal{K}$. Define $H_{l}^{E^{\prime} / \mathcal{F}}$ etc. in the same way, computed with respect to the differentials on $E^{\prime}, E^{\prime \prime}, E^{\prime \prime \prime}$ that pull back to $\omega$ under the corresponding 2-isogenies.

If $p=2$, then $(-1)^{\left[\mathcal{K}: \mathbb{Q}_{2}\right]} w(E / \mathcal{K})$ is given by

$$
\begin{array}{ll}
H_{2}^{E / \mathcal{K}} H_{2}^{E^{\prime} / \mathcal{K}} H_{2}^{E^{\prime \prime} / \mathcal{K}} H_{2}^{E^{\prime \prime \prime} / \mathcal{K}} & \text { if } d=1, \\
H_{2}^{E / \mathcal{K}} H_{2}^{E^{\prime} / \mathcal{K}} H_{2}^{E^{\prime} / \mathcal{F}} H_{2}^{E^{\prime \prime} / \mathcal{F}} & \text { if } d=2, \\
H_{2}^{E / \mathcal{F}} H_{2}^{E^{\prime} / \mathcal{F}} & \text { if } d=3, \\
H_{2}^{E / \mathcal{L}^{\prime}} H_{2}^{E^{\prime} / \mathcal{L}^{\prime}} H_{2}^{E / \mathcal{F}} H_{2}^{E^{\prime} / \mathcal{F}} H_{3}^{E / \mathcal{K}\left(\sqrt{\Delta_{E}}\right)} H_{3}^{E / \mathcal{F}} & \text { if } d=6 .
\end{array}
$$

If $p \neq 2$, the same formulae compute $w(E / \mathcal{K})$.

Acknowledgements. We would like to thank A. Bartel, D. Cain, B. D. Kim and A. J. Scholl for many helpful discussions. The first author is supported by a Royal Society University Research Fellowship.

1.3. Notation. Number fields are denoted by $K, F$, etc., and local fields of characteristic 0 by $\mathcal{K}, \mathcal{F}$, etc.; $F / K$ and $\mathcal{F} / \mathcal{K}$ are usually Galois extensions. The product $\prod_{v} \cdots$ always refers to a product over all places $v$ of $K$.

$|a|_{\mathcal{K}} \quad$ normalised absolute value of $a$ in $\mathcal{K}$.

$(a, \mathcal{F} / \mathcal{K})$ Artin symbol of $a$ in $\mathcal{F} / \mathcal{K}$. In all our cases it will have order 1 or 2 in $\operatorname{Gal}(\mathcal{F} / \mathcal{K})$, and we will regard it as \pm 1 .

$(a, b)_{\mathcal{K}} \quad$ Hilbert symbol in $\mathcal{K}($ the same as $(a, \mathcal{K}(\sqrt{b}) / \mathcal{K}))$.

$\langle\cdot, \cdot\rangle \quad$ usual inner product of (characters of $)$ representations. 
Notation for an elliptic curve $E / K$ or $E / \mathcal{K}$ :

$\operatorname{rk} E / K \quad$ Mordell-Weil rank of $E / K$, i.e. the rank of $E(K) /$ torsion. $\mathcal{X}_{p}(E / K) \quad \operatorname{Hom}_{\mathbb{Z}_{p}}\left(\lim _{\operatorname{Sel}_{p^{n}}}(E / K), \mathbb{Q}_{p} / \mathbb{Z}_{p}\right) \otimes \mathbb{Q}_{p}$, the dual $p^{\infty}$-Selmer. $\operatorname{rk}_{p} E / K \quad \operatorname{dim}_{\mathbb{Q}_{p}} \overrightarrow{\mathcal{X}_{p}}(E / K)$, the $p^{\infty}$-Selmer rank of $E / K$ $\left(=\operatorname{rk} E / K+\right.$ number of copies of $\mathbb{Q}_{p} / \mathbb{Z}_{p}$ in $\left.\amalg(E / K)\right)$. $w(E / k) \quad$ local root number of $E / k$ for $k$ local, or global root number $\prod_{v} w\left(E / k_{v}\right)$ for $k$ a number field. $w(E / k, \tau) \quad$ local/global root number for the twist of $E$ by $\tau$ (see [21]). $C(E / \mathcal{K}, \omega)=c(E / \mathcal{K}) \cdot\left|\omega / \omega^{\circ}\right|_{\mathcal{K}}$ for $\mathcal{K}$ non-Archimedean, where $c$ is the local Tamagawa number, and $\omega^{\circ}$ a Néron differential; $=\int_{E(\mathcal{K})}|\omega|$ for $\mathcal{K}=\mathbb{R}$ and $2 \int_{E(\mathcal{K})} \omega \wedge \bar{\omega}$ for $\mathcal{K}=\mathbb{C}$. ( $\omega$ is a non-zero invariant differential for $E / \mathcal{K}$.)

$C_{E / K} \quad \prod_{v} C\left(E / K_{v}, \omega\right)$ for any global invariant differential $\omega \neq 0$; independent of the choice of $\omega$ by the product formula.

$E_{\alpha} \quad$ quadratic twist of $E$ by $\alpha$.

For the definition and standard facts about root numbers we refer to [27], [21] and the appendix to [6]. The latter also contains a summary of basic properties of $\mathcal{X}_{p}(E / K)$ that we occassionally use.

\section{2-PARITY OVER TOTALLY REAL FIELDS}

There is a large supply of elliptic curves over number fields for which the $p$-parity conjecture is known to hold. Over $\mathbb{Q}$ it is true for all elliptic curves (4] Thm. 1.4), and for odd $p$ for elliptic curves with non-integral $j$-invariant over totally real fields ([20] Thm. 1). The purpose of this section is to extend the latter result to $p=2$ (Theorem 2.4). Its proof goes along similar lines to that of Monsky [19] for elliptic curves over $\mathbb{Q}$ and uses potential modularity as in [20].

We refer to Wintenberger [31] for the definition of modularity for elliptic curves over totally real fields. The two propositions below are known and are only included for completeness (see Taylor's [28] proof of Thm. 2.4 and [29] proof of Cor. 2.2).

Proposition 2.1. Let $F / K$ be a cyclic extension of totally real fields, and $E / K$ an elliptic curve. If $E / F$ is modular, then so is $E / K$.

Proof. Let $\pi$ be the cuspidal automorphic representation associated to $E / F$. Pick a generator $\sigma$ of $\operatorname{Gal}(F / K)$. Then $\pi^{\sigma}=\pi$ and therefore by Langlands' base change theorem $\pi$ descends to a cuspidal automorphic representation $\Pi$ over $K$. Associated to $\Pi$ there is a compatible system of representations $\rho_{\Pi, \lambda}$ (see [31]). Let $\rho=\rho_{\Pi, \lambda}$ be one of these. Its restriction to $\operatorname{Gal}(\bar{F} / F)$ agrees with $V=V_{l}(E / F) \otimes_{\mathbb{Q}_{l}} \mathbb{Q}_{\lambda}$. Because $E$ cannot have complex multiplication over $F$ (it is totally real), $V_{l}(E / F)$ is absolutely irreducible, and therefore the only representations that restrict to $V$ are $W=V_{l}(E / K) \otimes_{\mathbb{Q}_{l}} \mathbb{Q}_{\lambda}$ and its twists by characters of $\operatorname{Gal}(F / K)$. Hence $\rho$ and $W$ differ by a 1-dimensional twist, whence $W$ is also automorphic and $E / K$ is modular. 
Proposition 2.2. Let $E$ be an elliptic curve over a totally real field $K$, and $p$ a prime number. If the p-parity conjecture for $E$ is true over every totally real extension of $K$ where $E$ is modular, then it is true for $E / K$.

Proof. Because $E$ is potentially modular (31] Thm. 1), there is a Galois totally real extension $F / K$ over which $E$ becomes modular. By Solomon's induction theorem, there are soluble subgroups $H_{i}<G=\operatorname{Gal}(F / K)$ and integers $n_{i}$, such that

$$
\mathbf{1}_{G}=\sum_{i} n_{i} \operatorname{Ind}_{H_{i}}^{G} \mathbf{1}_{H_{i}}
$$

where 1 denotes the trivial character.

Write $K_{i}$ for $F^{H_{i}}$. Since $\operatorname{Gal}\left(F / K_{i}\right)=H_{i}$ is soluble, a repeated application of Proposition 2.1 shows that $E / K_{i}$ is modular. By Artin formalism for $L$-functions,

$$
L(E / K, s)=\prod_{i} L\left(E / K_{i}, s\right)^{n_{i}}
$$

On the other hand, writing $\mathcal{X}=\mathcal{X}_{p}(E / F)$, for every $H<G$ we have

$$
\mathrm{rk}_{p} E / K^{H}=\operatorname{dim} \mathcal{X}^{H}=\left\langle\mathcal{X}, \mathbf{1}_{H}\right\rangle_{H}=\left\langle\mathcal{X}, \operatorname{Ind}_{H}^{G} \mathbf{1}_{H}\right\rangle_{G} .
$$

The first equality is a standard fact about the $p^{\infty}$-Selmer group, see e.g. [4] Lemma 4.14, and the last one is Frobenius reciprocity.

By assumption, the $p$-parity conjecture holds for $E / K_{i}$. Therefore

$$
\begin{aligned}
\operatorname{rk}_{p} E / K & =\left\langle\mathcal{X}, \mathbf{1}_{G}\right\rangle_{G}=\sum_{i} n_{i} \operatorname{rk}_{p} E / K_{i} \\
& \equiv \sum_{i} n_{i} \operatorname{ord}_{s=1} L\left(E / K_{i}, s\right)=\operatorname{ord}_{s=1} L(E / K, s) \quad \bmod 2 .
\end{aligned}
$$

Remark 2.3. The proof also shows that $L(E / K, s)$ has a meromorphic continuation to $\mathbb{C}$, with the expected functional equation. This is the same argument as in [29], proof of Cor. 2.2.

Theorem 2.4. Let $K$ be a totally real field, and $E / K$ an elliptic curve with non-integral $j$-invariant. Then the p-parity conjecture holds for $E / K$ for every prime $p$.

Proof. For odd $p$ this is [20] Thm. 1, so suppose $p=2$. Let $\mathfrak{p}$ be a prime of $K$ with $\operatorname{ord}_{\mathfrak{p}} j(E)<0$. If $E$ has additive reduction at $\mathfrak{p}$, it becomes multiplicative over some totally real quadratic extension $K(\sqrt{\alpha})$, and the quadratic twist $E_{\alpha} / K$ has multiplicative reduction at $\mathfrak{p}$ as well. Because

$$
\begin{aligned}
w(E / K(\sqrt{\alpha})) & =w(E / K) w\left(E_{\alpha} / K\right) \\
\mathrm{rk}_{2} E / K(\sqrt{\alpha}) & =\mathrm{rk}_{2} E / K+\mathrm{rk}_{2} E_{\alpha} / K,
\end{aligned}
$$

it suffices to prove the theorem for elliptic curves with a prime of multiplicative reduction. Since multiplicative reduction remains multiplicative in all extensions, by Proposition 2.2 we may also assume that $E$ is modular.

By Friedberg-Hoffstein's theorem [10 Thm. B, there is a quadratic extension $K(\sqrt{\beta})$ of $K$ which is split at all bad primes for $E$ and such that 
the quadratic twist $E_{\beta}$ has analytic rank $\leq 1$. Since $E_{\beta}$ also has a prime of multiplicative reduction, by Zhang's theorem ([32] Thm. A) $\amalg\left(E_{\beta}\right)$ is finite and the Mordell-Weil rank of $E_{\beta} / K$ agrees with its analytic rank; in particular, the 2-parity conjecture holds for $E_{\beta} / K$. As it also holds for $E / K(\sqrt{\beta})$ by Kramer-Tunnell's theorem (see Cor. 4.6 below for a precise statement), it is true for $E / K$.

\section{Continuity of LOCAl invariants}

We gather a few basic facts concerning realisations of local fields as completions of totally real fields and continuity of standard invariants.

Lemma 3.1. Let $\mathcal{F} / \mathcal{K} / \mathbb{Q}_{p}$ be finite extensions, with $\mathcal{F} / \mathcal{K}$ Galois. There is a Galois extension of totally real number fields $F / K$ and a place $v_{0}$ of $K$ with a unique place $w_{0}$ of $F$ above it, such that $\mathcal{K}_{v_{0}} \cong \mathcal{K}$ and $\mathcal{F}_{w_{0}} \cong_{\mathcal{K}} \mathcal{F}$.

Proof. Say $\mathcal{K}=\mathbb{Q}_{p}[x] /(f)$ with monic $f \in \mathbb{Q}_{p}[x]$. If $\tilde{f} \in \mathbb{Q}[x]$ is monic and $p$-adically close enough to $f$, it defines the same extension of $\mathbb{Q}_{p}$ by Krasner's lemma. Now pick such an $\tilde{f}$ which is also $\mathbb{R}$-close to any polynomial in $\mathbb{R}[x]$ whose roots are real (weak approximation), and set $K_{0}=\mathbb{Q}[x] /(\tilde{f})$ and $\mathfrak{p}$ to be the prime above $p$ in $K_{0}$.

Pick a monic irreducible polynomial $g \in \mathcal{K}[x]$ whose splitting field is $\mathcal{F}$, and again approximate it $\mathfrak{p}$-adically with monic $\tilde{g} \in K_{0}[x]$ all of whose roots are totally real. Now let $F=K_{0}[x] /(\tilde{g})$ and let $K$ be the fixed field of $F$ under the decomposition group of some prime $w_{0}$ above $\mathfrak{p}$.

Lemma 3.2. For a local field $\mathcal{K}$ of characteristic 0 , the Hilbert symbol $(a, b)_{\mathcal{K}}$ is a continuous function of $a, b \in \mathcal{K}^{*}$.

Proof. Fix $a_{0}, b_{0} \in \mathcal{K}^{*}$, and set $\mathcal{F}=\mathcal{K}\left(\sqrt{a_{0}}\right)$. Then $\mathcal{K}(\sqrt{a}) \cong \mathcal{F}$ for $a$ in an open neighbourhood $V$ of $a_{0}$ in $\mathcal{K}^{*}$. The subgroup of norms $U=N_{\mathcal{F}} / \mathcal{K}^{\mathcal{F}^{*}}$ is open in $\mathcal{K}^{*}$, and $(a, b)_{\mathcal{K}}=\left(a_{0}, b_{0}\right)_{\mathcal{K}}$ for $a \in V, b \in b_{0} U$.

Proposition 3.3. Let $\mathcal{K}$ be a finite extension of $\mathbb{Q}_{p}$ and $E / \mathcal{K}$ an elliptic curve in Weierstrass form,

$$
E: y^{2}+a_{1} x y+a_{3} y=x^{3}+a_{2} x^{2}+a_{4} x+a_{6}, \quad a_{i} \in \mathcal{K} .
$$

There is an $\epsilon>0$ such that changing the $a_{i}$ to any $a_{i}^{\prime}$ with $\left|a_{i}-a_{i}^{\prime}\right|_{\mathcal{K}}<\epsilon$ does not change the conductor, minimal discriminant, Tamagawa number, $C\left(E, \frac{d x}{2 y+a_{1} x+a_{3}}\right)$, the root number of $E$ and $T_{l} E$ as a $\operatorname{Gal}(\overline{\mathcal{K}} / \mathcal{K})$-module for any given $l \neq p$.

Proof. The assertion for the conductor, minimal discriminant, Tamagawa number and $C$ follows from Tate's algorithm ([26] or [24] IV.9). The claim for $T_{l} E$ is a result of Kisin ([13] p. 569), and the root number is a function of $V_{l} E=T_{l} E \otimes \mathbb{Q}_{l}$. Alternatively, that the root number is locally constant can be proved in a more elementary way: see Helfgott's [12] Prop. 4.2 when $E$ has potentially good reduction; in the potentially multiplicative case it follows from Rohrlich's formula ([21] Thm. 2(ii)). 


\section{The Kramer-Tunnell Conjecture}

In this section we recall the Kramer-Tunnell theory of local norm indices, and prove the outstanding case of the conjecture of [16] for local fields of characteristic 0 (Theorem 4.7).

Notation 4.1. For a separable quadratic (or trivial) extension $\mathcal{F} / \mathcal{K}$ of local fields and an elliptic curve $E / \mathcal{K}$, write

$$
\kappa(E, \mathcal{F} / \mathcal{K})=(-1)^{\operatorname{dim}_{\mathbb{F}_{2}}\left(E(\mathcal{K}) / N_{\mathcal{F} / \mathcal{K}} E(\mathcal{F})\right)},
$$

where $N_{\mathcal{F} / \mathcal{K}}$ is the norm (or trace) map on points.

Remark 4.2. Alternatively, suppose $\mathcal{K}$ is non-Archimedean with char $\mathcal{K} \neq 2$, and that $\mathcal{F}=\mathcal{K}(\sqrt{\alpha})$ is a proper extension of $\mathcal{K}$. Write $E$ and its quadratic twist $E_{\alpha}$ in simplified Weierstrass form,

$$
E: y^{2}=x^{3}+a x^{2}+b x+c, \quad E_{\alpha}: y^{2}=x^{3}+\alpha a x^{2}+\alpha^{2} b x+\alpha^{3} c .
$$

By [16] Thm. 7.6,

$$
\kappa(E, \mathcal{F} / \mathcal{K})=(-1)^{\operatorname{ord}_{2} C\left(E / \mathcal{K}, \frac{d x}{y}\right) C\left(E_{\alpha} / \mathcal{K}, \frac{d x}{y}\right) / C\left(E / \mathcal{F}, \frac{d x}{y}\right) .}
$$

In particular, by Proposition 3.3, $\kappa$ is a locally constant function of the coefficients $a, b, c$ of $E$.

Theorem 4.3 (Kramer [15] Thm. 1). Let E/K be an elliptic curve over a number field, and $F / K$ a quadratic extension. Then

$$
(-1)^{\mathrm{rk}_{2} E / F}=\prod_{v} \kappa\left(E, F_{w} / K_{v}\right),
$$

where the product is taken over the places $v$ of $K$, and $w$ is any place of $F$ above $v$.

Conjecture 4.4 (Kramer-Tunnell [16] Conj. 3.1). Let $\mathcal{K}$ be a local field and $E / \mathcal{K}$ an elliptic curve. Let $\mathcal{F} / \mathcal{K}$ be a separable quadratic extension, and write $\chi: \operatorname{Gal}(\mathcal{F} / \mathcal{K}) \rightarrow\{ \pm 1\}$ for the non-trivial character. Let $\Delta_{E} \in \mathcal{K}^{*}$ be the discriminant of some model of $E / \mathcal{K}$. Then

$$
w(E / \mathcal{K}) w(E / \mathcal{K}, \chi)\left(-\Delta_{E}, \mathcal{F} / \mathcal{K}\right) \stackrel{?}{=} \kappa(E, \mathcal{F} / \mathcal{K}) .
$$

Theorem 4.5 (Kramer-Tunnell [16] p.315, §4, §5, §6, §8). The conjecture holds when $\mathcal{K}$ is Archimedean, when $E / \mathcal{K}$ has potentially multiplicative reduction, when $\mathcal{K}$ has odd residue characteristic, when $\mathcal{F} / \mathcal{K}$ is unramified, and when $E / \mathcal{K}$ has good reduction or reduction type IV or $\mathrm{IV}^{*}$.

Corollary 4.6 (cf. [16] p. 351). Let E be an elliptic curve over a number field $K$, and $F / K$ a quadratic extension. Then

$$
(-1)^{\mathrm{rk}_{2} E / F}=w(E / F),
$$

provided $F / K$ is unramified at those primes $v \mid 2$ of $K$ where $E$ has additive potentially good reduction not of type IV, IV*. 
Proof. Let $\chi: \operatorname{Gal}(F / K) \rightarrow\{ \pm 1\}$ be the non-trivial character. For a place $v$ of $K$ fix any $w \mid v$ in $F$, and write $\chi_{v}$ for the restriction of $\chi$ to $\operatorname{Gal}\left(F_{w} / K_{v}\right)$. Observe that Conjecture 4.4 also holds when $\mathcal{F}=\mathcal{K}$ and $\chi=1$. Thus,

$$
\begin{array}{rll}
(-1)^{\mathrm{rk}_{2} E / F} & \stackrel{4.3}{=} \prod_{v} \kappa\left(E, F_{w} / K_{v}\right) \\
& \stackrel{4.5}{=} \prod_{v} w\left(E / K_{v}\right) w\left(E / K_{v}, \chi_{v}\right)\left(-\Delta_{E}, F_{w} / K_{v}\right) \\
= & w(E / K) w(E / K, \chi) \cdot 1 \\
= & w(E / F),
\end{array}
$$

where the third equality is the product formula for the global root number and for Artin symbols.

Theorem 4.7. The Kramer-Tunnell conjecture holds over every local field $\mathcal{K}$ of characteristic 0.

Proof. By Theorem 4.5, we may suppose $\mathcal{K}$ is non-Archimedean. Let $\mathcal{E} / \mathcal{K}$ be the elliptic curve for which we want to prove the conjecture.

Pick a totally real field $K$ with completion $\mathcal{K}$ at some place $v_{0}$ (Lemma 3.1). Choose also a quadratic extension $F=K(\sqrt{\alpha}) / K$ with completion $\mathcal{F}$ above $v_{0}$ such that all places $v \neq v_{0}$ above 2 split in $F / K$ (weak approximation).

Let $E$ be an elliptic curve over $K$ that is sufficiently close to $\mathcal{E}$ at $v_{0}$, so that $w(\mathcal{E} / \mathcal{K})=w(E / \mathcal{K}), w\left(\mathcal{E}_{\alpha} / \mathcal{K}\right)=w\left(E_{\alpha} / \mathcal{K}\right),\left(-\Delta_{\mathcal{E}}, \mathcal{F} / \mathcal{K}\right)=\left(-\Delta_{E}, \mathcal{F} / \mathcal{K}\right)$ and $\kappa(\mathcal{E}, \mathcal{F} / \mathcal{K})=\kappa(E, \mathcal{F} / \mathcal{K})$ (Proposition 3.3, Lemma 3.2 and Remark 4.2). Moreover, we may assume that at some place $v_{1}$ of $K$ which is split in $F / K$ the curve $E$ has multiplicative reduction (weak approximation again). By construction, it suffices to prove the claim for $E / K_{v_{0}}$.

By the Kramer-Tunnell theorem 4.5, the terms in the two products

$\prod_{v}(-1)^{\operatorname{dim}_{\mathbb{F}_{2}} \frac{E\left(K_{v}\right)}{N E\left(K_{v}(\sqrt{\alpha})\right)}}$ and $\prod_{v} w\left(E / K_{v}\right) w\left(E_{\alpha} / K_{v}\right)\left(-\Delta_{E}, K_{v}(\sqrt{\alpha}) / K_{v}\right)$

agree except possibly at $v_{0}$. The first product multiplies to $(-1)^{\mathrm{rk}_{2} E / F}$ by Theorem 4.3. The second multiplies to $w(E / K) w\left(E_{\alpha} / K\right)$ which is also $(-1)^{\mathrm{rk}_{2} E / F}$ by Theorem 2.4 applied to $E / K$ and $E_{\alpha} / K$. So the contributions from $v_{0}$ are also equal.

Corollary 4.8. Let $E$ be an elliptic curve over a number field $K$, and $F / K$ a quadratic extension. Then

$$
(-1)^{\mathrm{rk}_{2} E / F}=w(E / F) .
$$

In other words, the 2-parity conjecture holds for $E / F$.

Proof. Same as the proof of Corollary 4.6.

Remark 4.9. As explained in [8, there are elliptic curves over number fields all of whose quadratic twists have the same root number. As predicted by the parity conjecture, we now find that these are precisely the curves all of whose quadratic twists have the same parity of the $2^{\infty}$-Selmer rank. In fact, for an elliptic curve $E$ over a number field $K$ the following conditions 
are equivalent:

(a) Every quadratic twist of $E / K$ has the same root number as $E$.

(b) $w(E / F)=w(E / K)^{[F: K]}$ for every finite extension $F / K$.

(c) Every quadratic twist of $E / K$ has the same parity of its $2^{\infty}$-Selmer rank as $E$.

(d) Every quadratic twist of $E / K$ has the same parity of the $\mathbb{F}_{2}$-dimension of its 2-Selmer group as $E$.

(e) $K$ has no real places, and $E$ acquires everywhere good reduction over some abelian extension of $K$.

(f) $K$ has no real places, and for all primes $p$ and all places $v \nmid p$ of $K$, the action of $\operatorname{Gal}\left(\bar{K}_{v} / K_{v}\right)$ on the Tate module $T_{p}(E)$ is abelian.

To be precise, (a) $\Leftrightarrow$ (c) follows from Corollary 4.8, (c) $\Leftrightarrow(\mathrm{d})$ holds because $E$ and its quadratic twists have the same 2-torsion, and the 2-primary part of $\amalg$ modulo its divisible part has square order; $(\mathrm{a}) \Leftrightarrow(\mathrm{b}) \Leftrightarrow(\mathrm{e}) \Leftrightarrow(\mathrm{f})$ is proved in [8] Thm. 1. Also, a slightly weaker form of $(\mathrm{a}) \Leftrightarrow(\mathrm{d})$ is proved in [18] $\S 9$.

\section{Elliptic CURVes With A p-ISOGENy}

The purpose of the section is to prove the $p$-parity conjecture for elliptic curves that admit a $K$-rational isogeny of degree $p$, for $p=2$ and 3 .

Notation 5.1. Let $\mathcal{K}$ be a local field of characteristic 0 . For an elliptic curve $E / \mathcal{K}$ with a $\mathcal{K}$-rational $p$-isogeny $\phi: E \rightarrow E^{\prime}$ write $\phi_{\mathcal{K}}: E(\mathcal{K}) \rightarrow E^{\prime}(\mathcal{K})$ for the induced map on $\mathcal{K}$-rational points, and define

$$
\sigma_{\phi}(E / \mathcal{K})=(-1)^{\operatorname{ord} p \frac{\# \operatorname{coker} \phi \mathcal{K}}{\# \operatorname{ker} \phi \mathcal{K}}}=(-1)^{\operatorname{ord}_{p} \frac{C\left(E^{\prime} / \mathcal{K}, \omega^{\prime}\right)}{C\left(E / \mathcal{K}, \phi^{*} \omega^{\prime}\right)}}
$$

for any invariant differential $\omega^{\prime}$ on $E^{\prime}$. (The second equality is an elementary computation using the usual filtration on $E(\mathcal{K})$; e.g. it is spelled out for abelian varieties in [23] Lemma 3.8.)

Theorem 5.2 (Cassels' formula). Let $K$ be a number field and $E / K$ an elliptic curve that admits a $K$-rational isogeny $\phi: E \rightarrow E^{\prime}$ of prime degree $p$. Then

$$
(-1)^{\mathrm{rk}_{p} E / K}=\prod_{v} \sigma_{\phi}\left(E / K_{v}\right) .
$$

Proof. Birch [1] p. 110, Fisher [9], Monsky [19] Cor. 2.8 or [4] Rmk. 4.4.

Conjecture 5.3. Let $\mathcal{K}$ be a local field of characteristic 0 and $E / \mathcal{K}$ an elliptic curve that admits a $\mathcal{K}$-rational isogeny $\phi: E \rightarrow E^{\prime}$ of prime degree $p$. Write $\mathcal{K}(\operatorname{ker} \phi)$ for the field obtained by adjoining the coordinates of points in $\operatorname{ker} \phi \subset E(\overline{\mathcal{K}})$ to $\mathcal{K}$. If $p \neq 2$, then

$$
w(E / \mathcal{K}) \stackrel{?}{=} \sigma_{\phi}(E / \mathcal{K}) \cdot(-1, \mathcal{K}(\operatorname{ker} \phi) / \mathcal{K}) .
$$

If $p=2$, then for any model of $E$ over $\mathcal{K}$ of the form $y^{2}=x^{3}+a x^{2}+b x$ with $\operatorname{ker} \phi=\{O,(0,0)\}$,

$$
w(E / \mathcal{K}) \stackrel{?}{=} \sigma_{\phi}(E / \mathcal{K}) \cdot \begin{cases}(a,-b)_{\mathcal{K}}\left(-2 a, a^{2}-4 b\right)_{\mathcal{K}}, & a \neq 0 \\ (-2,-b)_{\mathcal{K}}, & a=0\end{cases}
$$


This conjecture is known in most cases:

Theorem 5.4 ([2] Thm. 2.7, [3] Thms. 5, 6, §7). Conjecture 5.3 holds if

(1) $\mathcal{K}$ is Archimedean,

(2) $\mathcal{K}$ has residue characteristic $l \neq p$,

(3) $\mathcal{K}$ has residue characteristic $l=p>3$, and either $E$ has potentially ordinary reduction or $E$ achieves semistable reduction after an abelian extension of $\mathcal{K}$,

(4) $\mathcal{K}$ has residue characteristic $l=p=3$, and $E$ is semistable.

$(5) \mathcal{K}$ has residue characteristic $l=p=2$, and $E$ has either good ordinary or multiplicative reduction.

Corollary 5.5. Let $K$ be a number field and $E / K$ an elliptic curve that admits a K-rational isogeny $\phi: E \rightarrow E^{\prime}$ of prime degree $p$. Assume that at primes $v \mid p$ of $K$, the curve $E / K_{v}$ satisfies the relevant condition of Theorem 5.4. Then

$$
(-1)^{\mathrm{rk}_{p} E / K}=w(E / K) .
$$

Proof. If $p=2$, choose a model for $E / K$ of the form $y^{2}=x^{3}+a x^{2}+b x$ with $\operatorname{ker} \phi=\{O,(0,0)\}$. Now combine Theorems 5.2 and 5.4. The product of Artin/Hilbert symbols over all places of $K$ is 1 by the product formula.

Now we prove the conjecture for $p=2$ and 3 by a continuity argument. First observe that for $p=2$ the Hilbert symbol term in Conjecture 5.3 is continuous:

Lemma 5.6. Let $\mathcal{K}$ be a local field of characteristic 0 . The function

$$
h(a, b)= \begin{cases}(a,-b)_{\mathcal{K}}\left(-2 a, a^{2}-4 b\right)_{\mathcal{K}}, & a \neq 0 \\ (-2,-b)_{\mathcal{K}}, & a=0 .\end{cases}
$$

is continuous for $a, b \in \mathcal{K}$ with $b \neq 0$ and $a^{2}-4 b \neq 0$.

Proof. For $a \neq 0$ this is Lemma 3.2, When $|a|_{\mathcal{K}}$ is small, writing $($,$) for (,)_{\mathcal{K}}$,

$$
\begin{aligned}
(a,-b)\left(-2 a, a^{2}-4 b\right) & =(a,-b)\left(-2 a, 1-\frac{a^{2}}{4 b}\right)(-2 a,-4 b) \\
& =(a,-b)(-2 a, \square)(a,-4 b)(-2,-4 b)=(-2,-b),
\end{aligned}
$$

because elements of $\mathcal{K}$ close to 1 are squares.

Theorem 5.7. Conjecture 5.3 holds for $p=2$ and $p=3$.

Proof. Any 2-isogeny $\phi: E \rightarrow E^{\prime}$ of elliptic curves over a field of characteristic not 2 or 3 has a model

$$
\begin{array}{lll}
E_{a, b} & : & y^{2}=x^{3}+a x^{2}+b x \\
E_{a, b}^{\prime} & : & y^{2}=x^{3}-2 a x^{2}+\left(a^{2}-4 b\right) x \\
\phi_{a, b}(x, y) & = & \left(x+a+b x^{-1}, y-b y x^{-2}\right) .
\end{array}
$$

Similarly, when $p=3$, there is a model

$$
\begin{array}{lll}
E_{a, b} & : & y^{2}=x^{3}+a(x-b)^{2} \\
E_{a, b}^{\prime} & : & y^{2}=x^{3}+a x^{2}+18 a b x+a b(16 a-27 b) \\
\phi_{a, b}(x, y) & = & \left(x-4 a b x^{-1}+4 a b^{2} x^{-3}, y+4 a b y x^{-2}-8 a b^{2} y x^{-3}\right) .
\end{array}
$$


Conversely, for any $a, b$ in the ground field the formulae do define a $p$-isogeny, provided the resulting curves are non-singular (equivalently if $a\left(a^{2}-4 b\right) \neq 0$ for $p=2$, and if $a b(4 a+27 b) \neq 0$ for $p=3)$.

Say our elliptic curves and the $p$-isogeny are in this form, with $a, b \in \mathcal{K}$. Pick a totally real field $K$ with completion $\mathcal{K}$ at a place $v_{0}$ (Lemma 3.1). If $A, B \in K$ are $v_{0}$-close to $a, b$, then $E_{A, B}$ and $E_{A, B}^{\prime}$ are non-singular, so they are elliptic curves over $K$ with a $p$-isogeny. By Lemma 5.6 and Proposition 3.3, all the terms in the conjectural formula are the same for $E_{A, B} / K_{v_{0}}$ as for $E_{a, b} / \mathcal{K}$.

Moreover, by the weak approximation theorem, we may choose $A, B$ so that the set of places where $E_{A, B}$ has multiplicative reduction is non-empty and includes all $v \neq v_{0}$ above $p$. To satisfy the latter, take for example $(A, B)$ to be $v$-close to $(22,-7)$ for $p=2$ and $(1,-12)$ for $p=3$. Because $E_{A, B}$ is defined over a totally real field and has non-integral $j$-invariant,

$$
\begin{aligned}
\prod_{v} w\left(E_{A, B} / K_{v}\right) & =w\left(E_{A, B} / K\right) \stackrel{\text { 2.4 }}{=}(-1)^{\mathrm{rk}_{p} E_{A, B} / K} \\
& \stackrel{5.2}{=} \prod_{v} \sigma_{\phi_{A, B}}\left(E_{A, B} / K_{v}\right) \\
= & \prod_{v} \sigma_{\phi_{A, B}}\left(E_{A, B} / K_{v}\right) \cdot\left(\begin{array}{c}
\text { Artin/Hilbert symbol } \\
\text { from Conjecture 5.3 }
\end{array}\right) .
\end{aligned}
$$

By Theorem 5.4, the terms at $v$ in the first and the last expression agree for all $v \neq v_{0}$, so they must agree at $v_{0}$ as well. Thus the conjecture holds for $E_{A, B} / K_{v_{0}}$ and hence for $E_{a, b} / \mathcal{K}$.

As in Corollary 5.5, we deduce:

Corollary 5.8. Let $p=2$ or 3 and $K$ a number field. The p-parity conjecture holds for every elliptic curve $E / K$ that admits a $K$-rational p-isogeny,

$$
(-1)^{\mathrm{rk}_{p} E / K}=(-1)^{\operatorname{ord}_{p} \frac{C_{E / K}}{C_{E^{\prime} / K}}}=w(E / K),
$$

where $E^{\prime} / K$ is the isogenous curve.

\section{PARITY FOR TWISTS COMING FROM BRAUER RELATIONS}

The principal result of this section (Theorem 6.7) is a slight extension of the results of [6] on the $p$-parity conjecture for twists of elliptic curves. First we illustrate it in the simplest possible setting, when the Galois group is the symmetric group $S_{3}$. In fact, this is the only case needed for the proof of Theorem 1.2 in 97 .

6.1. Example: Galois group $G \cong \mathrm{S}_{3}$. As in $\$ 4$ and $\$ 5$, we begin with a formula expressing a Selmer rank in terms of local data. Recall [4] Thm. 4.11 (with $p=3$ ): 2

\footnotetext{
2 The contributions from $v \mid \infty$ to $\frac{C_{E / F} C_{E / K}{ }^{2}}{C_{E / M} C_{E / L}{ }^{2}}$ cancel when using the same $K$-rational $\omega$ over each field. The definition of $C$ in 4 excludes infinite places, so the formula there does not need the $\frac{C_{E / K^{2}}}{C_{E / L^{2}}}$ term, as it is then a rational square.
} 
Theorem 6.1. Let $F / K$ be an $\mathrm{S}_{3}$-extension of number fields, $M$ and $L$ intermediate fields of degree 2 and 3 over $K$, and $E / K$ an elliptic curve. Then

$$
\mathrm{rk}_{3} E / K+\mathrm{rk}_{3} E / M+\mathrm{rk}_{3} E / L \equiv \operatorname{ord}_{3} \frac{C_{E / F} C_{E / K}{ }^{2}}{C_{E / M} C_{E / L}{ }^{2}} \quad \bmod 2 .
$$

We want to prove the parity conjecture for the left-hand side. In other words, we claim that

$\left(\dagger_{\text {glo }}\right) \quad w(E / K) w(E / M) w(E / L)=(-1)^{\operatorname{ord}_{3} \frac{C_{E / F} C_{E / K}{ }^{2}}{C_{E / M} C_{E / L} L^{2}}}$.

Both sides are products of local terms, and, as in the Kramer-Tunnell case and the isogeny case, we want to compare the contributions above each place $v$ of $K$. It is elementary to check the following (see e.g. [4] proof of Prop. 3.3):

Fact 6.2. The contributions to each side of $t_{\text {glo }}$ are trivial whenever $v$ splits in $F$.

The remaining case when $v$ does not split in $F$ leads to the following purely local problem:

Theorem 6.3. Let $\mathcal{F} / \mathcal{K}$ be an $\mathrm{S}_{3}$-extension of $p$-adic fields, $\mathcal{M}$ and $\mathcal{L}$ intermediate fields of degree 2 and 3 over $\mathcal{K}$, and $\mathcal{E} / \mathcal{K}$ an elliptic curve. Then

$$
w(\mathcal{E} / \mathcal{K}) w(\mathcal{E} / \mathcal{M}) w(\mathcal{E} / \mathcal{L})=(-1)^{\operatorname{ord}_{3} \frac{C(\mathcal{E} / \mathcal{F}, \omega)}{C(\mathcal{E} / \mathcal{M}, \omega)}} .
$$

for any invariant differential $\omega$ for $E / \mathcal{K}$.

Proof. The formula is brutally worked out in [4] Prop. 3.3, except for the case when $\mathcal{K}$ has residue characteristic 2 or $3, \mathcal{E}$ has additive reduction and $\mathcal{F} / \mathcal{K}$ is ramified.

Now we use a continuity argument to settle this remaining case. Pick an $\mathrm{S}_{3}$-extension $F / K$ of totally real number fields with completions $K_{v_{0}}=\mathcal{K}$ and $F_{v_{0}}=\mathcal{F}$ for some prime $v_{0}$ of $K$ (Lemma 3.1). Choose an elliptic curve $E / K$ which is close enough $v_{0}$-adically to $\mathcal{E}$, with semistable reduction at all $v \neq v_{0}$ above 2 and 3 and at least one prime of multiplicative reduction. By 'close enough' we mean that the left- and the right-hand sides of ( $f_{\text {loc }}$ are the same for $E$ and $\mathcal{E}$ (Proposition 3.3); note that the right-hand side is independent of the choice of $\omega$.

By the 3-parity conjecture for $E$ over the intermediate fields of $F / K$ (Thm. 2.4) and Theorem 6.1, we find that (†, holds. Since the terms in it agree above all $v \neq v_{0}$ by 6.2 and the known cases of the formula, they must agree at $v_{0}$ as well. This proves $\dagger_{\text {loc }}$ for $E / K_{v_{0}}$ and hence for $\mathcal{E} / \mathcal{K}$ as well.

As a corollary of 6.2 and 6.3, the formula $\left(\dagger_{\text {glo }}\right.$ holds for all elliptic curves in all $\mathrm{S}_{3}$-extensions of number fields $F / K$. Combining it with Theorem 6.1. we get 
Theorem 6.4. Let $F / K$ be an $\mathrm{S}_{3}$-extension of number fields, and let $M$ and $L$ be intermediate fields of degree 2 and 3 over $K$, respectively. For every elliptic curve over $K$,

$$
(-1)^{\mathrm{rk}_{3} E / K+\mathrm{rk}_{3} E / M+\mathrm{rk}_{3} E / L}=(-1)^{\operatorname{ord}_{3} \frac{C_{E / F} C_{E / K}{ }^{2}}{C_{E / M} C_{E / L^{2}}}}=w(E / K) w(E / M) w(E / L) .
$$

6.2. General case. Now suppose $E / K$ is an elliptic curve, and $F / K$ any Galois extension of number fields with Galois group $G$ (generalising $G=\mathrm{S}_{3}$ above). The dual $p^{\infty}$-Selmer group $\mathcal{X}_{p}(E / F)$ is a $\mathbb{Q}_{p} G$-representation, and the $p$-parity conjecture for twists asserts that

$$
(-1)^{\left\langle\tau, \mathcal{X}_{p}(E / F)\right\rangle}=w(E / K, \tau),
$$

for every self-dual (complex) representation $\tau$ of $G$. There are twists for which the left-hand side can be expressed as a product of local terms, and one may hope to prove the conjecture for them by a place-by-place comparison.

This is what is done in [6] for a class of such twists, except that, as usual, the worst reduction cases are not considered there. Knowing the $p$-parity conjecture over totally real fields allows us to remove the constraints on the reduction for some of these twists, via a continuity argument.

After recalling the set $\mathbf{T}_{\Theta, p}$ of twists for which [6] gives a local $p$-parity formula, we carry out the continuity argument (Theorem 6.7 and Corollary 6.8). We end with a list of examples and applications in $\$ 6.3$.

Let $G$ be a finite group, and write $R_{G}$ for its (complex) representation ring. Write $\mathcal{H}$ for the set of subgroups of $G$ up to conjugacy; its elements are in one-to-one correspondence with transitive $G$-sets via $H \mapsto G / H$. The Burnside ring of $G$ is the free abelian group $\mathbb{Z} \mathcal{H}$, and the map $H \mapsto \mathbb{C}[G / H]$ extends by linearity to a natural map $\mathbb{Z} \mathcal{H} \longrightarrow R_{G}$. Following [6], elements in its kernel are called $G$-relations. Thus $\Theta=\sum n_{i} H_{i}$ is a $G$-relation if

$$
\bigoplus_{i} \mathbb{C}\left[G / H_{i}\right]^{\oplus n_{i}} \cong 0
$$

as a virtual representation.

Fix a prime $p$ and an identification $\overline{\mathbb{Q}}_{p} \cong \mathbb{C}$. For a self-dual $\mathbb{Q}_{p} G$-representation $\rho$ and a $G$-relation $\Theta$, define the regulator constant

$$
\mathcal{C}_{\Theta}^{\mathbb{Q}_{p}}(\rho)=\mathcal{C}_{\Theta}(\rho)=\prod_{i} \operatorname{det}\left(\frac{1}{|H|}\langle,\rangle \mid \rho^{H}\right)^{n_{i}} \in \mathbb{Q}_{p}^{*} / \mathbb{Q}_{p}^{* 2},
$$

where $\langle$,$\rangle is any non-degenerate G$-invariant $\overline{\mathbb{Q}}_{p}$-valued bilinear pairing on $\rho$, and $\operatorname{det}(\langle\rangle \mid V,) \operatorname{denotes} \operatorname{det}\left(\left\langle v_{i}, v_{j}\right\rangle_{i, j}\right)$ for any basis $v_{i}$ of $V$. By [6] $\S 2.2, \mathcal{C}_{\Theta}(\rho)$ is a well-defined element of $\mathbb{Q}_{p}^{*} / \mathbb{Q}_{p}^{* 2}$ and is independent of $\langle$,$\rangle . We define$

$$
\mathbf{T}_{\Theta, p}=\left\{\begin{array}{c}
\text { self-dual } \overline{\mathbb{Q}}_{p} G \text {-representations } \tau \text { such that } \\
\langle\tau, \rho\rangle \equiv \operatorname{ord}_{p} \mathcal{C}_{\Theta}(\rho) \bmod 2 \\
\text { for all self-dual } \mathbb{Q}_{p} G \text {-representations } \rho .
\end{array}\right\}
$$

With the identification $\overline{\mathbb{Q}}_{p} \cong \mathbb{C}$, we may consider $\mathbf{T}_{\Theta, p} \subset R_{G}$. 
Let $F / K$ be a Galois extension of local or global fields with Galois group $G$, and $E / K$ an elliptic curve. For a $G$-relation $\Theta=\sum n_{i} H_{i}$ for brevity we write

$$
C(E, \Theta)=\prod_{i} C\left(E / F^{H_{i}}, \omega\right)^{n_{i}}
$$

for any invariant differential $\omega$ for $E / K$. Finally, if $K$ is a number field, we can decompose

$$
C(E, \Theta)=\prod_{v} C_{w \mid v}(E, \Theta)
$$

where $v$ runs over the places of $K$, and $C_{w \mid v}(E, \Theta)$ picks all terms in the product defining $C(E, \Theta)$ from all places above $v$. (It is not hard to see that $C(E, \Theta)$ in the local case and $C_{w \mid v}(E, \Theta)$ in the number field case are independent of the choice of $\omega$, see e.g. [6], proof of Cor. 3.4.)

According to [5] Thms. 1.1, 1.5 (or [6] Thm. 1.14),

Theorem 6.5. Let $F / K$ be a Galois extension of number fields with Galois group $G, p$ a prime and $\Theta$ a G-relation. For every elliptic curve $E / K$, the $\mathbb{Q}_{p} G$-representation $\mathcal{X}_{p}(E / F)$ is self-dual, and

$$
\left\langle\tau, \mathcal{X}_{p}(E / F)\right\rangle \equiv \operatorname{ord}_{p} C(E, \Theta) \quad \bmod 2 \quad \text { for all } \tau \in \mathbf{T}_{\Theta, p} .
$$

In other words, the quotient of the 'fudge factors' over the fields defined by $\Theta$ computes the parity of $\tau$ in the Selmer group.

Notation 6.6. Write $B_{G}$ for the subgroup of $R_{G}$ generated by the image of the Burnside ring and all representations of the form $\sigma \oplus \sigma^{*}$.

Theorem 6.7. Let $\mathcal{F} / \mathcal{K}$ be a Galois extension of local fields of characteristic 0 with Galois group $G$. Let $\mathcal{E} / \mathcal{K}$ be an elliptic curve, $p$ a prime and $\Theta$ a $G$-relation. If $\mathcal{E}$ is semistable or $\mathcal{K}$ has residue characteristic $\geq 5$, then

$$
w(\mathcal{E}, \tau)=(-1)^{\operatorname{ord}_{p} C(\mathcal{E}, \Theta)} \quad \text { for all } \quad \tau \in \mathbf{T}_{\Theta, p} .
$$

In all cases, the formula holds for $\tau \in \mathbf{T}_{\Theta, p} \cap B_{G}$.

Proof. The first claim is a special case of [6] Cor. 3.3. Suppose therefore that $\tau \in \mathbf{T}_{\Theta, p} \cap B_{G}$.

Let $F / K$ be a Galois extension of totally real fields and $v_{0}$ a prime of $K$, such that $K_{v_{0}}=\mathcal{K}$ and $\operatorname{Gal}(F / K)=\operatorname{Gal}(\mathcal{F} / \mathcal{K})$ (Lemma 3.1). Let $E / K$ be an elliptic curve with non-integral $j$-invariant, which is semistable at all primes $v \neq v_{0}$ above 2 and 3 , and $E$ is sufficiently close to $\mathcal{E}$. By sufficiently close we mean that $E / K_{v_{0}}$ and $\mathcal{E} / \mathcal{K}$ have the same local root numbers, valuations of minimal discriminants and local Tamagawa numbers in all intermediate fields of $\mathcal{F} / \mathcal{K}$ (possible by Proposition 3.3).

By [6] Cor. 3.4,

$$
w\left(E / K_{v}, \operatorname{Res}_{G_{v}} \tau\right)=(-1)^{\operatorname{ord}_{p} C_{w \mid v}(E, \Theta)} \quad \text { for all } v \neq v_{0} .
$$

Taking the product over all places $v$ we get that the asserted formula at $v_{0}$ (and hence the claim for $\mathcal{E} / \mathcal{K}$ ) is equivalent to the global formula

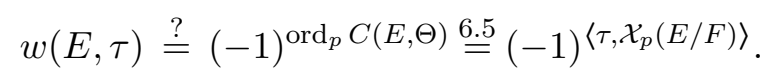


But this is true, because $\tau \cong\left(\sigma \oplus \sigma^{*}\right) \oplus \bigoplus_{i} \mathbb{C}\left[G / H_{i}\right]^{\oplus n_{i}} \in B_{G}$, and

$$
w\left(E, \sigma \oplus \sigma^{*}\right)=1=(-1)^{\left\langle\sigma \oplus \sigma^{*}, \mathcal{X}_{p}(E / F)\right\rangle}
$$

by self-duality ([6] Prop. A.2(1)), and

$$
w\left(E, \mathbb{C}\left[G / H_{i}\right]\right)=w\left(E / F^{H_{i}}\right)=(-1)^{\mathrm{rk}_{p} E / F^{H_{i}}}=(-1)^{\left\langle\mathbb{C}\left[G / H_{i}\right], \mathcal{X}_{p}(E / F)\right\rangle}
$$

by inductivity (6] Prop. A.2(2)) and the p-parity conjecture for $E$ over the totally real field $F^{H_{i}}$ (Theorem 2.4).

Corollary 6.8. Let $F / K$ be a Galois extension of number fields with Galois group $G$. Let $E / K$ be an elliptic curve, $p$ a prime and $\Theta$ a $G$-relation. Then

$$
w(E, \tau)=(-1)^{\operatorname{ord}_{p} C(E, \Theta)}=(-1)^{\left\langle\tau, \mathcal{X}_{p}(E / F)\right\rangle}
$$

for all $\tau \in \mathbf{T}_{\Theta, p}$ such that $\operatorname{Res}_{G_{v}} \tau \in B_{G_{v}}$ for every prime $v \mid 6$ where $E$ has additive reduction. 3 .

Proof. The first equality follows by the same argument as the proof of Cor. 3.4 of [6]. (Essentially take the product over all places, and check that everything behaves well under the passage to decomposition groups.) The second equality is Theorem 6.5.

Remark 6.9. We expect Theorem 6.7 and Corollary 6.8 to hold for all $\tau \in \mathbf{T}_{\Theta, p}$, irrespectively of the reduction type of $E$.

\subsection{Examples and applications.}

Example 6.10. The group $G=\mathrm{S}_{3}$ has four subgroups up to conjugacy, $H=\{1\}, \mathrm{C}_{2}, \mathrm{C}_{3}, G$ and three irreducible representations: 1 (trivial), $\epsilon$ (sign) and $\rho$ (2-dimensional). There is a $G$-relation

$$
\Theta=\{1\}-2 \mathrm{C}_{2}-\mathrm{C}_{3}+2 G,
$$

unique up to multiples. One easily checks that $\mathcal{C}_{\Theta}^{\mathbb{Q}_{p}}(\mathbf{1})=\mathcal{C}_{\Theta}^{\mathbb{Q}_{p}}(\epsilon)=\mathcal{C}_{\Theta}^{\mathbb{Q}_{p}}(\rho)=3$, independently of the prime $p$. Thus,

$$
\mathbf{1} \oplus \epsilon \oplus \rho \in \mathbf{T}_{\Theta, 3} .
$$

This representation lies in the Burnside ring of $G$,

$$
\mathbb{C}\left[G / \mathrm{C}_{2}\right] \oplus \mathbb{C}\left[G / \mathrm{C}_{3}\right] \ominus \mathbb{C}[G / G]=(\mathbf{1} \oplus \rho) \oplus(\mathbf{1} \oplus \epsilon) \ominus \mathbf{1}=\mathbf{1} \oplus \epsilon \oplus \rho .
$$

By Corollary 6.8, we find that in an $\mathrm{S}_{3}$-extension of number fields $F / K$ and an elliptic curve $E / K$,

$$
w(E, \mathbf{1} \oplus \epsilon \oplus \rho)=(-1)^{\operatorname{ord}_{3} C(E, \Theta)}=(-1)^{\left\langle\mathbf{1} \oplus \epsilon \oplus \rho, \mathcal{X}_{p}(E / F)\right\rangle} .
$$

Noting that

$$
w(E, \mathbf{1} \oplus \epsilon \oplus \rho)=w(E / K) w\left(E / F^{\mathrm{C}_{3}}\right) w\left(E / F^{\mathrm{C}_{2}}\right)
$$

and similarly for the Selmer rank by inductivity ([6] Prop. A.2(2)), we recover Theorem 6.4. In the same way, Theorem 6.7 recovers Theorem 6.3.

\footnotetext{
${ }^{3}$ In particular, this always applies to $\tau \in \mathbf{T}_{\Theta, p} \cap B_{G}$.
} 
Example 6.11. Let $G=\mathrm{D}_{2 p}$ be the dihedral group of order $2 p$ with $p \equiv 3 \bmod 4$. As for $\mathrm{S}_{3}$, there is a $G$-relation

$$
\Theta=\{1\}-2 \mathrm{C}_{2}-\mathrm{C}_{p}+2 \mathrm{D}_{2 p} .
$$

The irreducible $\mathbb{C} G$-representations are $\mathbf{1}$ (trivial), $\epsilon(\operatorname{sign})$ and $\sigma_{1}, \ldots, \sigma_{\frac{p-1}{2}}$ (2-dimensional). The irreducible $\mathbb{Q}_{p} G$-representations are $\mathbf{1}, \epsilon$ and $\rho=\bigoplus_{i}{ }^{2} \sigma_{i}$, and their regulator constants are (cf. [6] Ex. 2.20)

$$
\mathcal{C}_{\Theta}^{\mathbb{Q}_{p}}(\mathbf{1})=\mathcal{C}_{\Theta}^{\mathbb{Q}_{p}}(\epsilon)=\mathcal{C}_{\Theta}^{\mathbb{Q}_{p}}(\rho)=p .
$$

Therefore $\tau=\mathbf{1} \oplus \epsilon \oplus \rho \in \mathbf{T}_{\Theta, p}$ (as $\frac{p-1}{2}$ is odd); it is also in the Burnside ring of $G$,

$$
\mathbb{C}\left[G / \mathrm{C}_{2}\right] \oplus \mathbb{C}\left[G / \mathrm{C}_{p}\right] \ominus \mathbb{C}[G / G]=(\mathbf{1} \oplus \rho) \oplus(\mathbf{1} \oplus \epsilon) \ominus \mathbf{1}=\tau .
$$

Now we apply Corollary 6.8 .

Proposition 6.12. Suppose $F / K$ is a Galois extension of number fields with Galois group $G \cong \mathrm{D}_{2 p}$ for a prime $p \equiv 3 \bmod 4$. Then for every elliptic curve $E / K$ and every 2-dimensional irreducible $\mathbb{C} G$-representation $\sigma$,

$$
w(E, \sigma \oplus \mathbf{1} \oplus \operatorname{det} \sigma)=(-1)^{\left\langle\sigma \oplus \mathbf{1} \oplus \operatorname{det} \sigma, \mathcal{X}_{p}(E / F)\right\rangle} .
$$

Proof. By Corollary 6.8 and the example above,

$$
w(E, \mathbf{1} \oplus \epsilon \oplus \rho)=(-1)^{\operatorname{ord}_{p} \frac{C_{E / F} C_{E / K}{ }^{2}}{\left(C_{E / F} C_{p}\right)\left(C_{E / F} \mathrm{C}_{2}\right)^{2}}}=(-1)^{\left\langle\mathbf{1} \oplus \epsilon \oplus \rho, \mathcal{X}_{p}(E / F)\right\rangle}
$$

with $\epsilon$ and $\rho$ as in the example. Now $\sigma$ is one of the constituents of $\rho$, and $\operatorname{det} \sigma=\epsilon$. Because $\mathcal{X}_{p}(E / F)$ is a $\mathbb{Q}_{p}$-rational representation,

$$
\left\langle\rho, \mathcal{X}_{p}(E / F)\right\rangle=\frac{p-1}{2}\left\langle\sigma, \mathcal{X}_{p}(E / F)\right\rangle .
$$

On the other hand, by equivariance of local (and therefore global) root numbers ([22] Thms. 1, 2),

$$
w(E, \rho)=w(E, \sigma)^{(p-1) / 2}=w(E, \sigma),
$$

and the result follows.

The proposition confirms [6] Hypothesis 4.1 for elliptic curves when $p \equiv 3 \bmod 4$. Thus, [6] shows:

Theorem 6.13. Let $K$ be a totally real number field, $p \equiv 3 \bmod 4$ a prime, and $E / K$ an elliptic curve. Assume either that $K=\mathbb{Q}$ or that $E$ has nonintegral $j$-invariant. Suppose $F$ is a p-extension of an abelian extension of $K$, Galois over K. Then

$$
(-1)^{\left\langle\tau, \mathcal{X}_{p}(E / F)\right\rangle}=w(E, \tau)
$$

for every orthogonal representation $\tau$ of $\operatorname{Gal}(F / K)$; in other words, the p-parity conjecture holds for the twist of $E$ by $\tau$. 
Proof. Combine [6] Thm. 4.5 with the above proposition and the $p$-parity theorems over $\mathbb{Q}$ and over totally real fields (4] Thm. 1.4 and [20] Thm. 1).

Theorem 6.14. Let $K$ be a number field, $p \equiv 3 \bmod 4$ a prime, and $E / K$ an elliptic curve. Suppose $F$ is a p-extension of a Galois extension $M / K$, Galois over $K$. If the p-parity conjecture

$$
(-1)^{\mathrm{rk}_{p} E / L}=w(E / L)
$$

holds for subfields $K \subset L \subset M$, then it holds for subfields $K \subset L \subset F$.

Proof. Combine [6] Thm. 4.3 with Proposition 6.12.

Theorem 6.15. For every elliptic curve $E / \mathbb{Q}$ and number field $L \subset \mathbb{Q}\left(E\left[3^{\infty}\right]\right)$,

$$
(-1)^{\mathrm{rk}_{3} E / L}=w(E / L) .
$$

Proof. Same argument as in 6] Ex. 4.8, using [6] Thm. 4.3 together with Proposition 6.12, 3-parity for elliptic curves with a 3-isogeny (Corollary 5.8) and over abelian extensions of $\mathbb{Q}([5]$ Thm. 1.2).

Another application of Corollary 6.8 is for general $p$ but $p$-adic towers where all orthogonal $\mathbb{C} G$-representations are in $B_{G}$. As an example, we may take a 'false Tate curve extension' over $\mathbb{Q}$ :

Example 6.16. Let $E / \mathbb{Q}$ be an elliptic curve, $p$ an odd prime, $m$ an integer which is not a perfect $p$ th power, and $F=\mathbb{Q}\left(\mu_{p^{n}}, \sqrt[p]{m}\right)$ for some $n \geq 1$. The complex irreducible representations of $G=\operatorname{Gal}(F / \mathbb{Q})$ are of the form $\chi \otimes \rho_{j}$ for a one-dimensional character $\chi$ of $\operatorname{Gal}\left(\mathbb{Q}\left(\mu_{p^{n}} / \mathbb{Q}\right)\right)$ and

$$
\rho_{0}=\mathbf{1}, \quad \rho_{j}=\mathbb{C}\left[G / H_{j}\right] \ominus \mathbb{C}\left[G / H_{j-1}\right] \quad(j=1, \ldots, n),
$$

where $H_{j}=\operatorname{Gal}\left(F / \mathbb{Q}\left(\sqrt[p^{j}]{m}\right)\right)$ is a subgroup of index $p^{j}$. The self-dual ones are the $\rho_{j}$ and the non-trivial character $\epsilon$ of $\operatorname{Gal}\left(\mathbb{Q}\left(\mu_{p}\right) / \mathbb{Q}\right)$ of order 2 , so all orthogonal representations of $G$ lie in $B_{G}$. Thus the $p$-parity conjecture holds for twists of $E$ by all $\tau \in \mathbf{T}_{\Theta, p}$ for every $G$-relation $\Theta$. Writing $U_{j}=\operatorname{Gal}\left(F / \mathbb{Q}\left(\mu_{p}, \sqrt[p]{m}\right)\right)$, one checks that

$$
\mathbf{1} \oplus \epsilon \oplus \rho_{j} \in \mathbf{T}_{U_{j}-U_{j-1}-(p-1) H_{j}+(p-1) H_{j-1}, p} .
$$

In particular, every orthogonal representation of $G$ is a sum of trivial, sign representations of $G$ and those in $\mathbf{T}_{\Theta, p}$ for some $\Theta$. Because the $p$-parity conjecture holds for $E / \mathbb{Q}$ and its quadratic twist by $\epsilon$, it follows that

$$
(-1)^{\left\langle\tau, \mathcal{X}_{p}(E / F)\right\rangle}=w(E, \tau)
$$

for every orthogonal representation $\tau$ of $G$. This example removes the semistability restriction from [4] Prop 4.13. See also [2] $\S 4$ for related results. 


\section{PARity over Fields AND The GLOBAl ROOT NUMBER FORMula}

Now we prove the two main results over number fields, the parity conjecture assuming finiteness of $\amalg$ and the formula for the global root number. The only two ingredients are the 2-isogeny theorem from $\$ 5$ and the $\mathrm{S}_{3}$ example from \$6] The argument is based on that of [4] Thm. 3.6.

Theorem 7.1. Let $E$ be an elliptic curve over a number field $K$, and suppose that $\amalg(E / K(E[2]))$ has finite 2- and 3-primary parts. Then

$$
(-1)^{\mathrm{rk} E / K}=w(E / K) \text {. }
$$

Proof. Write $F=K(E[2])$, and note that $\operatorname{Gal}(F / K) \subset \mathrm{GL}_{2}\left(\mathbb{F}_{2}\right) \cong \mathrm{S}_{3}$. By assumption, the 2- and 3-primary parts of $\amalg(E / k)$ are finite for $K \subset k \subset F$, see e.g. 4] Rmk. 2.10.

If $E$ has a $K$-rational 2-torsion point, the result follows from Corollary 5.8 , If $F / K$ is cubic, then the formula follows from that over $F$, as both the parity of the rank and the root number are unchanged in odd degree Galois extensions (see e.g. 6] Prop. A.2(3)).

The remaining case is $\operatorname{Gal}(F / K) \cong \mathrm{S}_{3}$. Let $M$ be the quadratic extension of $K$ in $F$, and let $L$ be one of the cubic ones. By the above argument,

$$
(-1)^{\mathrm{rk} E / M}=w(E / M) \quad \text { and } \quad(-1)^{\mathrm{rk} E / L}=w(E / L) \text {. }
$$

On the other hand, by Theorem 6.4

$$
(-1)^{\mathrm{rk} E / K+\mathrm{rk} E / M+\mathrm{rk} E / L}=w(E / K) w(E / M) w(E / L) .
$$

The construction gives an explicit formula for the global root number:

Theorem 7.2. Let $E$ be an elliptic curve over a number field $K$. Write $F=K(E[2])$ and $G=\operatorname{Gal}(F / K)$. Choose a non-trivial 2-torsion point $P$ of $E$, defined over $K$ if $G=\mathrm{C}_{2}$. Write $E^{\prime}=E /\{O, P\}$ for the 2-isogenous curve defined over $L=K(P)$. Then

$$
w(E / K)= \begin{cases}(-1)^{\operatorname{ord}_{2} \frac{C_{E / L} C_{E / F}}{C_{E^{\prime} / L} C_{E^{\prime} / F}}+\operatorname{ord}_{3} \frac{C_{E / F} C_{E / K}{ }^{2}}{C_{E / K\left(\sqrt{\Delta_{E}} C_{E / L} C^{2}\right.}}} & G=\mathrm{S}_{3}, \\ (-1)^{\operatorname{ord}_{2} \frac{C_{E / L}}{C_{E^{\prime} / L}}} & G \neq \mathrm{S}_{3} .\end{cases}
$$

Proof. When $G=\mathrm{C}_{1}$ or $\mathrm{C}_{2}$, apply the 2-isogeny theorem (Corollary 5.8). If $G=\mathrm{C}_{3}$, then $w(E / K)=w(E / L)([6]$ Prop. A.2(3)), and the result follows from that for $E / L$. Finally, suppose $G=\mathrm{S}_{3}$. Noting that $K\left(\sqrt{\Delta_{E}}\right)$ is the quadratic extension of $K$ in $F$,

$$
\begin{array}{ll}
(-1)^{\operatorname{ord} 2 \frac{C_{E / L} C_{E / F}}{C_{E^{\prime} / L} C_{E^{\prime} / F}}} & =w(E / L) w(E / F), \\
(-1)^{\operatorname{ord} 3 \frac{C_{E / F} C_{E / K}{ }^{2}}{C_{E / K\left(\sqrt{\Delta_{E}}\right)} C_{E / L}{ }^{2}}} & =w(E / L) w(E / F) w(E / K),
\end{array}
$$

by the 2 -isogeny theorem over $L$ and $F$ and by Theorem 6.4 , respectively. 
Remark 7.3. It is already implicit in [4 that, assuming finiteness of $\amalg$, $(-1)^{\mathrm{rk} E / K}$ is given by the formula in the right-hand side of Theorem 7.2 , It only relies on Cassels' formula 5.2 and Theorem 6.1 and bypasses all comparisons with root numbers.

\section{The local Root number formula}

From the proof of the global root number theorem, with a bit of extra work, one can also extract a formula for the local root numbers (Theorem 8.4 below). The idea is to write the right-hand side of Theorem 7.2 as a product of local terms and compare them to the local root numbers. This is slightly delicate, as these local terms, denoted by $\mathfrak{m}(E / \mathcal{K})$ below, must not depend on the global extension $K(E[2]) / K$. Also, to get a manageable relation between $w(E / \mathcal{K})$ and $\mathfrak{m}(E / \mathcal{K})$ we will make the construction symmetric in the three non-trivial 2-torsion points of $E$.

Notation 8.1. Let $k$ be a field (later local or global) and $E$ an elliptic curve over $k$. Write $S=E[2] \backslash\{O\}$, the set of non-trivial 2-torsion points of $E / \bar{k}$. The symmetric group $\mathrm{S}_{3}$ acts on $S$. Denote the irreducible representations of $\mathrm{S}_{3}$ by 1 (trivial), $\epsilon$ (sign) and $\rho$ (2-dimensional).

For a group $G$ acting on $S$ (later a Galois group) write $\operatorname{Orb}_{G} S$ for the set of $G$-orbits of $S$. Abusing notation, we will write $P \in \operatorname{Orb}_{G} S$ for a representative of the corresponding orbit, and $\mathbf{1}, \epsilon, \rho$ for the restrictions of these representations to $G$. (Note that $\rho$ may become reducible.)

A point $P \in S$ gives rise to a 2-isogeny $\phi: E \rightarrow E /\{O, P\}$, and we write $\sigma_{P}$ for $\sigma_{\phi}$ as defined in Notation 5.1 .

Definition 8.2. Let $\mathcal{K}$ be a local field of characteristic 0 and $E / \mathcal{K}$ an elliptic curve. Set $\mathcal{M}=\mathcal{K}\left(\sqrt{\Delta_{E}}\right), \mathcal{F}=\mathcal{K}(E[2]), G=\operatorname{Gal}(\mathcal{F} / \mathcal{K}), d=[\mathcal{F}: \mathcal{K}]$ and

$$
\begin{aligned}
\mathfrak{m}^{1}(E / \mathcal{K}) & =\prod_{P \in \operatorname{Orb}_{G} S} \sigma_{P}(E / \mathcal{K}(P)), \\
\mathfrak{m}^{1 \epsilon}(E / \mathcal{K}) & =\prod_{P \in S}\left\{\begin{array}{ll}
\sigma_{P}(E / \mathcal{F}), & 2 \mid d \\
1, & 2 \nmid d
\end{array},\right. \\
\mathfrak{m}^{1 \epsilon \rho}(E / \mathcal{K}) & = \begin{cases}(-1)^{\operatorname{ord} 3} \frac{C(E / \mathcal{F}, \omega)}{C(E / \mathcal{M}, \omega)}, & d=6 \\
1, & d \neq 6,\end{cases} \\
\mathfrak{m}(E / \mathcal{K}) & =\mathfrak{m}^{\mathbf{1} \rho}(E / \mathcal{K}) \mathfrak{m}^{1 \epsilon}(E / \mathcal{K}) \mathfrak{m}^{1 \epsilon \rho}(E / \mathcal{K}) .
\end{aligned}
$$

Remark 8.3. The $\mathfrak{m}$ compute various Selmer ranks of twists. If $E$ is defined over a number field $K$, and $\mathbf{1}, \epsilon$ and $\rho$ are the representations of $G=\operatorname{Gal}(K(E[2]) / K) \subset \mathrm{S}_{3}$ as in 8.1, then

$$
\begin{aligned}
\prod_{v} \mathfrak{m}^{1 \rho}\left(E / K_{v}\right) & =(-1)^{\left\langle\mathbf{1} \oplus \rho, \mathcal{X}_{2}(E / F)\right\rangle}, \\
\prod_{v} \mathfrak{m}^{1 \epsilon}\left(E / K_{v}\right) & =(-1)^{\left\langle\mathbf{1} \oplus \epsilon, \mathcal{X}_{2}(E / F)\right\rangle}, \\
\prod_{v} \mathfrak{m}^{1 \epsilon \rho}\left(E / K_{v}\right) & =(-1)^{\left\langle\mathbf{1} \oplus \epsilon \oplus \rho, \mathcal{X}_{3}(E / F)\right\rangle} .
\end{aligned}
$$


This follows from the theorem below (combined with Corollary 5.8 and Theorem (6.4), although it can also be deduced directly from Cassels' formula (Theorem 5.2) and Theorem 6.1

Theorem 8.4. Let $\mathcal{K}$ be a local field of characteristic zero, $E / \mathcal{K}$ an elliptic curve, and $\mathbf{1}, \epsilon, \rho$ the representations of $G=\operatorname{Gal}(\mathcal{K}(E[2]) / \mathcal{K})$ as in 8.1. Then

$$
\begin{aligned}
& \mathfrak{m}^{1 \rho}(E / \mathcal{K})=w(E / \mathcal{K}) w(E / \mathcal{K}, \rho)\left(-1,-\Delta_{E}\right) \mathcal{K} \\
& \mathfrak{m}^{1 \epsilon}(E / \mathcal{K})=w(E / \mathcal{K}) w(E / \mathcal{K}, \epsilon)\left(-1, \Delta_{E}\right) \mathcal{K}, \\
& \mathfrak{m}^{1 \epsilon \rho}(E / \mathcal{K})=w(E / \mathcal{K}) w(E / \mathcal{K}, \epsilon) w(E / \mathcal{K}, \rho)
\end{aligned}
$$

In particular,

$$
\mathfrak{m}(E / \mathcal{K}) \quad=(-1,-1) \mathcal{K} w(E / \mathcal{K}) .
$$

Proof. Write $\mathcal{F}=\mathcal{K}(E[2])$. We will use $\operatorname{Ind}_{\mathcal{L} / \mathcal{K}}$ as a shorthand for $\operatorname{Ind}_{\operatorname{Gal}(\mathcal{F} / \mathcal{L})}^{\operatorname{Gal}(\mathcal{K})}$. Recall that local root numbers satisfy 'self-duality', 'inductivity in degree 0 ' and the 'determinant formula': for a representation $\tau$ of $G$ and $\mathcal{K} \subset \mathcal{L} \subset \mathcal{F}$,

$$
\begin{aligned}
& w(E / \mathcal{K}, \tau)=\overline{w\left(E / \mathcal{K}, \tau^{*}\right)} \\
& w(E / \mathcal{L})=w\left(\operatorname{Ind}_{\mathcal{L} / \mathcal{K}} \mathbf{1}\right)^{2} w\left(E / \mathcal{K}, \operatorname{Ind}_{\mathcal{L} / \mathcal{K}} \mathbf{1}\right) \\
& w\left(\tau \oplus \tau^{*}\right)=(\operatorname{det} \tau)(-1)
\end{aligned}
$$

where $(\operatorname{det} \tau)(-1)$ is the character $\operatorname{det} \tau$ evaluated at the image of -1 under the local reciprocity map. These are all well-known properties of root numbers; see e.g. [6] App. A.

(1) Choose a Weierstrass model $y^{2}=f(x)$ for $E / \mathcal{K}$. For $P=(r, 0) \in S$, define $a_{P}, b_{P} \in \mathcal{K}(P)$ by $f(x+r)=x^{3}+a_{P} x^{2}+b_{P} x$. Note that

$$
1 \oplus \rho=\bigoplus_{P \in \operatorname{Orb}_{G} S} \operatorname{Ind}_{\mathcal{K}(P) / \mathcal{K}} \mathbf{1}
$$

Taking all products below over $P \in \operatorname{Orb}_{G} S$, we have

$$
\begin{aligned}
& \mathfrak{m}^{\mathbf{1} \rho}(E / \mathcal{K})=\prod \sigma_{P}(E / \mathcal{K}(P)) \\
& \stackrel{5.7}{=} \prod(E / \mathcal{K}(P)) \begin{cases}\left(a_{P},-b_{P}\right)_{\mathcal{K}(P)}\left(-2 a_{P}, a_{P}^{2}-4 b_{P}\right)_{\mathcal{K}(P)}, & a_{P} \neq 0 \\
\left(-2,-b_{P}\right)_{\mathcal{K}(P)}, & a_{P}=0\end{cases} \\
& \stackrel{8.6}{=}(-1,-1)_{\mathcal{K}} \prod w(E / \mathcal{K}(P)) \\
& \stackrel{(\text { Ind) }}{=}(-1,-1)_{\mathcal{K}} \prod w\left(\operatorname{Ind}_{\mathcal{K}(P) / \mathcal{K}} \mathbf{1}\right)^{2} w\left(E / \mathcal{K}, \operatorname{Ind}_{\mathcal{K}(P) / \mathcal{K}} \mathbf{1}\right) \\
& =(-1,-1)_{\mathcal{K}} w(\mathbf{1} \oplus \rho)^{2} w(E, \mathbf{1} \oplus \rho) \\
& \stackrel{\text { det) }}{=}(-1,-1)_{\mathcal{K}} \in(-1) w(E, \mathbf{1} \oplus \rho) \text {. }
\end{aligned}
$$

(2) If $[\mathcal{F}: \mathcal{K}]$ is odd, then $\mathbf{1}=\epsilon$ and the statement trivially holds. Otherwise, by (Ind) the right-hand side of the asserted formula is $w(E / \mathcal{M})$, where $\mathcal{M}=\mathcal{K}\left(\sqrt{\Delta_{E}}\right)$ is the quadratic extension of $\mathcal{K}$ in $\mathcal{F}$. This is the same as $w(E / \mathcal{F})$ because $\operatorname{Gal}(\mathcal{F} / \mathcal{M})$ is either $\mathrm{C}_{1}$ or $\mathrm{C}_{3}$ (use (Ind), (*) and (det)). On the other hand, by Theorem 5.7 and Lemma 8.6 the left-hand side is $w(E / \mathcal{F})^{3}(-1,-1)_{\mathcal{F}}$. This is indeed $w(E / \mathcal{F})$, as $[\mathcal{F}: \mathcal{K}]$ is even and so $(-1,-1)_{\mathcal{F}}=1$ by Remark 8.5 below. 
(3) If $[\mathcal{F}: \mathcal{K}]<6$, then the left-hand side is trivial, and the right-hand side is of the form $w\left(E / \mathcal{K}, V \oplus V^{*}\right)$, which is also 1 by $(*)$. If $[\mathcal{F}: \mathcal{K}]=6$, let $\mathcal{M}$ and $\mathcal{L}$ be a quadratic and a cubic extension of $\mathcal{K}$ in $\mathcal{F}$. Then

$$
\begin{gathered}
\mathfrak{m}^{1 \epsilon \rho}(E / \mathcal{K})=(-1)^{\operatorname{ord} 3} \frac{C(E / \mathcal{F})}{C(E / \mathcal{M})} \stackrel{6.3}{=} w(E / \mathcal{K}) w(E / \mathcal{M}) w(E / \mathcal{L}) \\
\stackrel{(\text { Ind })}{=} w(E, \mathbf{1} \oplus \epsilon \oplus \rho) w(\mathbf{1} \oplus \epsilon \oplus \rho)^{2} \stackrel{(\text { det })}{=} w(E, \mathbf{1} \oplus \epsilon \oplus \rho) .
\end{gathered}
$$

Remark 8.5. Recall that $(-1,-1)_{\mathcal{K}}$ measures whether the algebra of Hamiltonian quaternions $\mathbb{Q}\langle 1, i, j, k\rangle$ is split or ramified over $\mathcal{K}$, so

$$
(-1,-1)_{\mathcal{K}}= \begin{cases}(-1)^{[\mathcal{K}: \mathbb{R}]} & \text { if } \mathcal{K} \text { is Archimedean, } \\ 1 & \text { if } \mathcal{K} \text { has odd residue characteristic } \\ (-1)^{\left[\mathcal{K}: \mathbb{Q}_{2}\right]} & \text { if } \mathcal{K} \text { has residue characteristic } 2\end{cases}
$$

In particular, Theorem 8.4 directly implies Theorem 1.12 ,

The following lemma was used in the proof of the theorem:

Lemma 8.6. Suppose $\mathcal{K}$ is a local field of characteristic 0 , and $f(x) \in \mathcal{K}[x]$ is monic of degree 3. Let $r_{1}, r_{2}, r_{3}$ be its roots in $\overline{\mathcal{K}}$, and $\mathcal{F}=\mathcal{K}\left(r_{1}, r_{2}, r_{3}\right)$. For $r \in\left\{r_{1}, r_{2}, r_{3}\right\}$ write $f(x+r)=x^{3}+a_{r} x^{2}+b_{r} x$. Then

$$
\prod_{r}\left\{\begin{array}{ll}
\left(a_{r},-b_{r}\right)_{\mathcal{K}(r)}\left(-2 a_{r}, a_{r}^{2}-4 b_{r}\right)_{\mathcal{K}(r)}, & a_{r} \neq 0 \\
\left(-2, b_{r}\right)_{\mathcal{K}(r)}, & a_{r}=0
\end{array}=(-1,-1)_{\mathcal{K}},\right.
$$

the product taken over some representatives of the orbits of $G=\operatorname{Gal}(\mathcal{F} / \mathcal{K})$ on $\left\{r_{1}, r_{2}, r_{3}\right\}$.

Proof. In view of Lemma 5.6, we can assume that all $a_{r} \neq 0$. Write $N$ for the norm map from $\mathcal{F}$ to $\mathcal{K}$. Recall that the Hilbert symbol $()=,(,)_{\mathcal{F}}$ is bilinear, symmetric, and satisfies

$$
\begin{array}{ll}
(a, 1-a)=(a,-a)=1, & \\
(a+b,-a b)=\left(1+\frac{b}{a},-\frac{b}{a}\right)(a,-a b)=(a, b), & \\
(a, b)(b, c)(a, c)(-1, a b c)=(-1,-1) & \text { if } a+b+c=0 \\
(a,-b)(b,-c)(c,-a)=(-1,-1) & \text { if } a+b+c=0 \\
(a, b)(-a,-c)(b,-c)=1, & \text { if } a+b+c=0 \\
(a, N b)_{\mathcal{K}}=(a, b) & \text { for } a \in \mathcal{K}, b \in \mathcal{F},
\end{array}
$$

whenever the constituents are non-zero.

Case 1: $\mathcal{F}=\mathcal{K}$. Say $r=r_{1}$. Then $a_{r}^{2}-4 b_{r}=\left(r_{2}-r_{3}\right)^{2}$ is a square in $\mathcal{K}$, so the second Hilbert symbol is trivial. The first one is

$$
\left(\left(r_{1}-r_{2}\right)+\left(r_{1}-r_{3}\right),-\left(r_{1}-r_{2}\right)\left(r_{1}-r_{3}\right)\right)=\left(r_{1}-r_{2}, r_{1}-r_{3}\right),
$$

and similarly for $r=r_{2}$ and $r_{3}$. The total product is

$$
\left(r_{1}-r_{2}, r_{1}-r_{3}\right) \cdot\left(r_{2}-r_{1}, r_{2}-r_{3}\right) \cdot\left(r_{3}-r_{1}, r_{3}-r_{2}\right)=(-1,-1) .
$$


Case 2: $r_{1} \in \mathcal{K}, r_{2}, r_{3} \notin \mathcal{K}$, and the product is taken over $r \in\left\{r_{1}, r_{2}\right\}$. Let $s=r_{2}-r_{1}, t=r_{3}-r_{1}$, and expand

$$
\begin{aligned}
& f\left(x+r_{1}\right)=x(x-s)(x-t)=x^{3}+(-s-t) x^{2}+s t x \\
& f\left(x+r_{2}\right)=x(x+s)(x+s-t)=x^{3}+(2 s-t) x^{2}+\left(s^{2}-s t\right) x .
\end{aligned}
$$

The total product of Hilbert symbols is

$$
\begin{aligned}
& (-s-t,-s t)_{\mathcal{K}} \cdot\left(2 s+2 t,(s-t)^{2}\right)_{\mathcal{K}} \cdot(s+(s-t),-s(s-t)) \cdot\left(-4 s+2 t, t^{2}\right)= \\
& \left(-s-t,-\frac{1}{4}\right)_{\mathcal{K}}(-s-t, N(2 s))_{\mathcal{K}} \cdot(2 s+2 t,-1)_{\mathcal{K}}(2 s+2 t, N(s-t))_{\mathcal{K}} \cdot(s, s-t) \cdot 1= \\
& (-s-t,-1)_{\mathcal{K}}(-s-t, 2 s)(2,-1)_{\mathcal{K}}(s+t,-1)_{\mathcal{K}}(2 s+2 t, s-t)(s, s-t)= \\
& (-1,-1)_{\mathcal{K}}(-s-t, 2 s)(s+t, s-t)(2, s-t)(s, s-t)= \\
& (-1,-1)_{\mathcal{K}}(-s-t, 2 s)(s+t, s-t)(2 s, s-t)=(-1,-1)_{\mathcal{K}} .
\end{aligned}
$$

Case 3: $[\mathcal{F}: \mathcal{K}]=3$. The claim follows from that for $f$ over $\mathcal{F}$, because $(-1,-1)_{\mathcal{K}}=(-1,-1)_{\mathcal{F}}$ and the terms in the left-hand side of the formula also remain the same for each $r$.

Case $4:[\mathcal{F}: \mathcal{K}]=6$. Invoke the formula over the quadratic and over a cubic intermediate field of $\mathcal{F} / \mathcal{K}$, and multiply the two.

\section{REFERENCES}

[1] B. J. Birch, Conjectures concerning elliptic curves, Proc. Sympos. Pure Math., Vol. VIII (1965), Amer. Math. Soc., Providence, R.I, 106-112.

[2] J. Coates, T. Fukaya, K. Kato, R. Sujatha, Root numbers, Selmer groups and noncommutative Iwasawa theory, 2007, to appear in J. Algebraic Geom.

[3] T. Dokchitser, V. Dokchitser, Parity of ranks for elliptic curves with a cyclic isogeny, J. Number Theory 128 (2008), 662-679.

[4] T. Dokchitser, V. Dokchitser, On the Birch-Swinnerton-Dyer quotients modulo squares, 2006, arxiv: math.NT/0610290, to appear in Annals of Math.

[5] T. Dokchitser, V. Dokchitser, Self-duality of Selmer groups, Math. Proc. Cam. Phil. Soc. 146 (2009), 257-267.

[6] T. Dokchitser, V. Dokchitser, Regulator constants and the parity conjecture, 2007, arxiv: 0709.2852, to appear in Invent. Math.

[7] T. Dokchitser, V. Dokchitser, Root numbers of elliptic curves in residue characteristic 2, Bull. London Math. Soc. 40 (2008), 516-524.

[8] T. Dokchitser, V. Dokchitser, Elliptic curves with all quadratic twists of positive rank, Acta Arith. 137 (2009), 193-197.

[9] T. Fisher, Appendix to V. Dokchitser, Root numbers of non-abelian twists of elliptic curves, Proc. London Math. Soc. (3) 91 (2005), 300-324.

[10] S. Friedberg, and J. Hoffstein, Nonvanishing theorems for automorphic L-functions on GL(2), Annals of Math. 142 (2), 1995, 385-423.

[11] E. Halberstadt, Signes locaux des courbes elliptiques en 2 et 3, C. R. Acad. Sci. Paris Série I Math. 326 (1998), no. 9, 1047-1052.

[12] H. A. Helfgott, On the behaviour of root numbers in families of elliptic curves, arXiv: math/0408141 3 .

[13] M. Kisin, Local constancy in $p$-adic families of Galois representations, Math. Z., 230 (1999), 569-593.

[14] S. Kobayashi, The local root number of elliptic curves with wild ramification, Math. Ann. 323 (2002), 609-623.

[15] K. Kramer, Arithmetic of elliptic curves upon quadratic extension, Trans. Amer. Math. Soc. 264 (1981), 121-135. 
[16] K. Kramer, J. Tunnell, Elliptic curves and local $\epsilon$-factors, Compos. Math. 46 (1982), 307-352.

[17] B. Mazur, K. Rubin, Finding large Selmer ranks via an arithmetic theory of local constants, Annals of Math. 166 (2), 2007, 579-612.

[18] B. Mazur, K. Rubin, Ranks of twists of elliptic curves and Hilbert's tenth problem, preprint, 2009, arxiv: 0904.3709.

[19] P. Monsky, Generalizing the Birch-Stephens theorem. I: Modular curves, Math. Z., 221 (1996), 415-420.

[20] J. Nekováŕ, On the parity of ranks of Selmer groups IV, to appear in Compos. Math.

[21] D. Rohrlich, Galois Theory, elliptic curves, and root numbers, Compos. Math. 100 (1996), 311-349.

[22] D. Rohrlich, Galois invariance of local root numbers, preprint, 2008, http://math.bu.edu/people/rohrlich/invariance.pdf.

[23] E. Schaefer, Class groups and Selmer groups, J. Number Theory 56 (1996), no. 1, $79-114$.

[24] J. H. Silverman, Advanced Topics in the Arithmetic of Elliptic Curves, GTM 151, Springer-Verlag 1994.

[25] J. Tate, On the conjectures of Birch and Swinnerton-Dyer and a geometric analog, Séminaire Bourbaki, 18e année, 1965/66, no. 306.

[26] J. Tate, Algorithm for determining the type of a singular fiber in an elliptic pencil, in: Modular Functions of One Variable IV, Lect. Notes in Math. 476, B. J. Birch and W. Kuyk, eds., Springer-Verlag, Berlin, 1975, 33-52.

[27] J. Tate, Number theoretic background, in: Automorphic forms, representations and L-functions, Part 2 (ed. A. Borel and W. Casselman), Proc. Symp. in Pure Math. 33 (AMS, Providence, RI, 1979) 3-26.

[28] R. Taylor, On icosahedral Artin representations II, American Journal of Mathematics 125 (2003), 549-566.

[29] R. Taylor, Remarks on the conjecture of Fontaine and Mazur, J. Inst. Math. Jussieu 1, 2002, 1-19.

[30] D. Whitehouse, Root numbers of elliptic curves over 2-adic fields, preprint, 2006, http://www-math.mit.edu/ dw/maths/elliptic2.pdf.

[31] J.-P. Wintenberger, Potential modularity of elliptic curves over totally real fields, appendix to 20 .

[32] S. Zhang, Heights of Heegner points on Shimura curves, Annals of Math. 153 (2001), $27-147$.

Robinson College, Cambridge CB3 9AN, United Kingdom

E-mail address: t.dokchitser@dpmms.cam.ac.uk

Gonville \& Caius College, Cambridge CB2 1TA, United Kingdom

E-mail address: v.dokchitser@dpmms.cam.ac.uk 